\title{
Indice de productivité des taillis-sous-futaie de chêne dans la région Centre
}

\author{
N. LE GOFF \\ avec la collaboration technique de L. GARROS et R. CANTA \\ I.N.R.A., Station de Sylviculture et de Production \\ Centre de Recherches forestières de Nancy, Champenoux, F 54280 Seichamps
}

\begin{abstract}
Résumé
Afin de connaîte el de mesurer laptitude pour la croissance de la production du chêne de stations portant actuellement des taillis-sous-futaie à base de chênes et souvent pauvres en réserves dans la région Centre, 17 couples «futaic/taillis-sous-futaie » de chêne ont été repérés sur le terrain; chaque couple se compose de 2 placettes, l'une établie en taillissous-futaie, l'autre en futaie, caractérisées par des conditions de station semblables.

Le peuplement de futaie de chaque couple a fourni un indice de productivité de référence, défini comme le rapport de la hauteur dominante du peuplement à celle au même âge donnée par la table de production à classe de fertilité unique établic pour le chêne rouvre en secteur ligérien. Les indices de productivité ainsi évalués permetuent de situer les 17 couples dans 3 classes de productivité (hauteur dominante ì 160 ans du peuplement de futaie comprise entre 28 et 36 mètres).
\end{abstract}

La recherche d'un indice de productivité pour les taillis-sous-futaic a été basée sur l'étude de la croissance en circonférence des arbres de réserve, s'appuyant sur des résultats antérieurs démontrant la bonne liaison existant avec la fertilité des stations. Il a de plus été tenté de rendre compte de l'effet de conditions de concurrence variées pour les réserves, dépendant en partie des traitements sylvicoles suivis, et qui peuvent être détectées et synthétisées par la mesure du rapport de la hauteur du houppicr à la hauteur totale actuelle des arbres. Enfin, il a été tenté, par l'échantillonnage réalisé (arbres pris parmi les plus vigoureux dans chaque classe d'âge des taillis-sous-futaie), et lors de la définition de l'indice de productivité, de prendre en compte la variabilité individuelle des arbres, différente d'un peuplement à l'autre, ne serait-ce qu'en regard des grandes variations du nombre d'arbres de réserve à l'hectarc.

L'indice de productivité défini pour les taiilis-sous-futaie de chêne a été identifié ì une mesure particulière de la distribution des valeurs du rapport de la circonférence observée des arbres de réserve échantillonnés à la circonférence estimée (à partir de la relation établie entre la circonférence à $1,30 \mathrm{~m}$, l'âge et la hauteur relative du houppier des arbres).

I. indice de productivité des taillis-sous-futaie de chêne se trouve lié par unc relation linéaire simple à l'indice de productivité délini pour les futaies. Il est renclu possible ainsi de comparer la productivité relative de stations portant des peuplements traités en taillis-sous-futaie et en futaie, done d'adopter un même classement vis-à-vis de l'aptitude des stations ì la croissance du chêne. II est possible aussi de prévớr, grâce à la table de production pour les futaics de chêre de cette région, le niveau de production en volume qu'aurait un peuplement de taillis-sous-futaie une fois converti en futaie, après détermination de sa productivité relative. 
L'application des résultats de cette étude à la détermination des productivités relatives des taillis-sous-futaie, fait l'objet d'une annexe à lat fin de l'article; les lecteurs intéressés y trouveront les renvois au texte nécessaires, notamment pour les relations de base et les graphiques à utiliser dans chaque cas particulicr.

\section{Introduction}

La mise en valeur des taillis et taillis-sous-futaic ${ }^{11)}$ de la moitié Nord de la France, jugés insuffisamment productifs et pour la plupart situés en forêt privée, fait l'objet depuis 1976 d'une action concertéc de la D.G.R.S.T. regroupant des laboratoires de II.N.R.A. (du C.N.R.F. en particulier) et des organismes représentant la forêt privée. La conversion ou la transformation de ces peuplements en futaies feuillues d'essences productrices de bois d'ouvre apparaît comme la solution optimale sur les stations les plus productives, et peut semble-t-il même être envisagée pour les petites propriétés grâce à une sylviculture appropriéc, qui reste cependant à définir. Ainsi, la détermination de la productivité de ces taillis et taillis-sous-futaie vis-à-vis de ces essences fcuillues apparaît-clle primordiale pour décider des choix à faire.

La conversion ou la transformation des taillis-sous-futaie de la région Centre (:) - les taillis sont exclus de l'étude - est envisagée bien sûr sur la base de la conservation du chêne en tant qu'essence principale, celle-ci étant l'essence constitutive de base (quelquefois à l'état pur) de la réserve de ces taillis-sous-futaic et fournissant par ailleurs le bois d’œuvre le plus recherché. L'évaluation de leur productivité pourra donc se faire de façon directe, basée sur la croissance relative des chênes réservés en taillis-sous-futaie et dépendant de la fertilité de la station; une étude similaire sur la productivité du frêne en région Nord-Picardie (LE Goff, 1982) a nécessité de relier un indice de productivité (évalué pour des peuplements de futaie) à des facteurs physiques de la station, le frêne neétant pas une essence aussi représentative de ces taillissous-futaie. Cette dernière méthode d'évaluation de la productivité pour une essence et une station données a été qualifiée $d$ ' «indirecte» par opposition à la première qui sera utilisée ici : cette dénomination a été utilisée par Carmean (1975) lors d'un inventaire des différentes méthodologies dévaluation de la productivité se rapportant à ces deux groupes de méthodes et mises largement en application aux U.S.A.

L'évaluation directe de la productivité pour une essence fait généralement appel à un indice, hauteur dominante à un âge de référence le plus souvent, indice que l'on peut relier à la production totale en volume à cet âge quand on dispose d'unc table de production ou des relations équivalentes pour la construire et liant la production à la hauteur et à l'âge, donnant alors la mesure réelle de la productivité d'une station. Cette mesure n'est toutefois pas indispensable dans le seul but du classement de stations et le souci de disposer, pour les taillis-sous-futaie à base de chênes de la région Centre, d’une mesure de productivité absolue (volume total à l'hectare produit à un

(1) On pourra se référer pour Ie jugement porté sur la situation actuelle de ces peuplements et leur devenir, aux documents fournis par les organismes contractants à la D.G.R.S.T. (en particulier à la «Demande d'Aide à la Recherche » $n^{\circ}$ 76-7-0193).

(2) Les taillis-sous-futaie de la région Centre situés en forêt privée couvrent, selon une estimation faite par le C.R.P.F. de cette région, $49 \%$ de la superficie boisée privée, soit environ 360000 hectares. 
âge donné par un peuplement de futaie de productivité comparable) a ainsi sans doute guidé les initiateurs ${ }^{(3)}$ de cette étude vers la recherche de «couples» futaie/taillissous-futaie sur «stations» comparables (4) de façon à convertir l'indice de productivité qui serait défini pour les taillis-sous-futaie en mesure de productivité réeile dans le cas de conversion en futaic ; la table de production établie par Pardé (1962) pour les futaies de chêne en secteur ligérien en donnerait alors la possibilité. Cette évaluation, en réalité, n'aura de sens pratique que pour des futaies de densité proche de celle de la table, futaies à durée de révolution trop longue pour qu'elles constituent un objectif à atteindre dans la majorité des forêts privées, surtout pour les moins étendues d'entre elles. Nous verrons cependant comment, en poursuivant la recherche dans cette voie, nous avons pu nous servir de la mesure de la productivité relative fournie par le peuplement de futaie de chaque couple pour, d’une part tester la mesure proposée de la productivité relative de ces taillis-sous-futaie, d'autre part fournir une comparaison possible de ces deux mesures, rendant ainsi la mesure relative proposée pour les taillissous-futaie de chêne plus démonstrative.

\section{Base de l'étude : les couples futaie/taillis-sous-futaie}

\subsection{Définition et principes de leur recherche}

Un «couple» futaic/tail'is-sous-futaie est défini comme lensemble de deux peuplements, l'un traité en futaie et l'autre en taillis-sous-futaie de même productivité potentielle vis-à-vis du chêne. La reconnaissance des couples a été basée sur l'identité de certains caractères de la station, caractères permettant de supposer des conditions de croissance équivalentes pour les chênes dans les deux types de peuplement (5) : la futaie et le taillis-sous-futaie de chaque couple ont été choisis si possible à proximité lun de l'autre (ils sont parfois adjacents) de manière à s'assurer de positions topographiques et de profils de sol voisins. Des sondages à la tarière pédologique en différents endroits de la futaie et du taillis-sous-futaie ont permis de contrôler la similitude des horizons du sol ainsi que celle de leur épaisseur. Les taillis-sous-futaie devaient par ailleurs comporter un nombre de réserves en chêne suffisant et la futaie constituer un peuplement plein et équienne. Le nombre relativement réduit de couples finalement retenus, 17 au total (voir tableau 1), tient essentiellement à la difficulté rencontrée de trouver à proximité l'un de l'autre futaie et taillis-sous-futaie, les peuplements de futaie étant plutôt situés en forêts domaniales et ceux de taillis-sous-futaie au contraire, en forêts privées ou communales.

(3) L'initiative de cette étude revient essentiellement à J. Bouchon, Maître de Recherches à la Station de Sylviculture et de Production et à M. Bonneal, Directeur de la Station des Sols forestiers au C.N.R.F.

(4) La «station» sera définic dans toute la suite par l'ensemble des caractères du milieu qui conditionnent la croissance de l'essence à laquelle on se réfère : elle a donc unc productivité bien définic vis-à-vis du chênc.

(5) La prospection pour la recherche de couples éventuels futaie/taillis-sous-futaic est due au C.R.P.F. de la région Centre, le contrôle de l'homogénéité des conditions de sol et de la topographie à M. Bonneau, Directeur de la Station des Sols forestiers au C.N.R.F. 


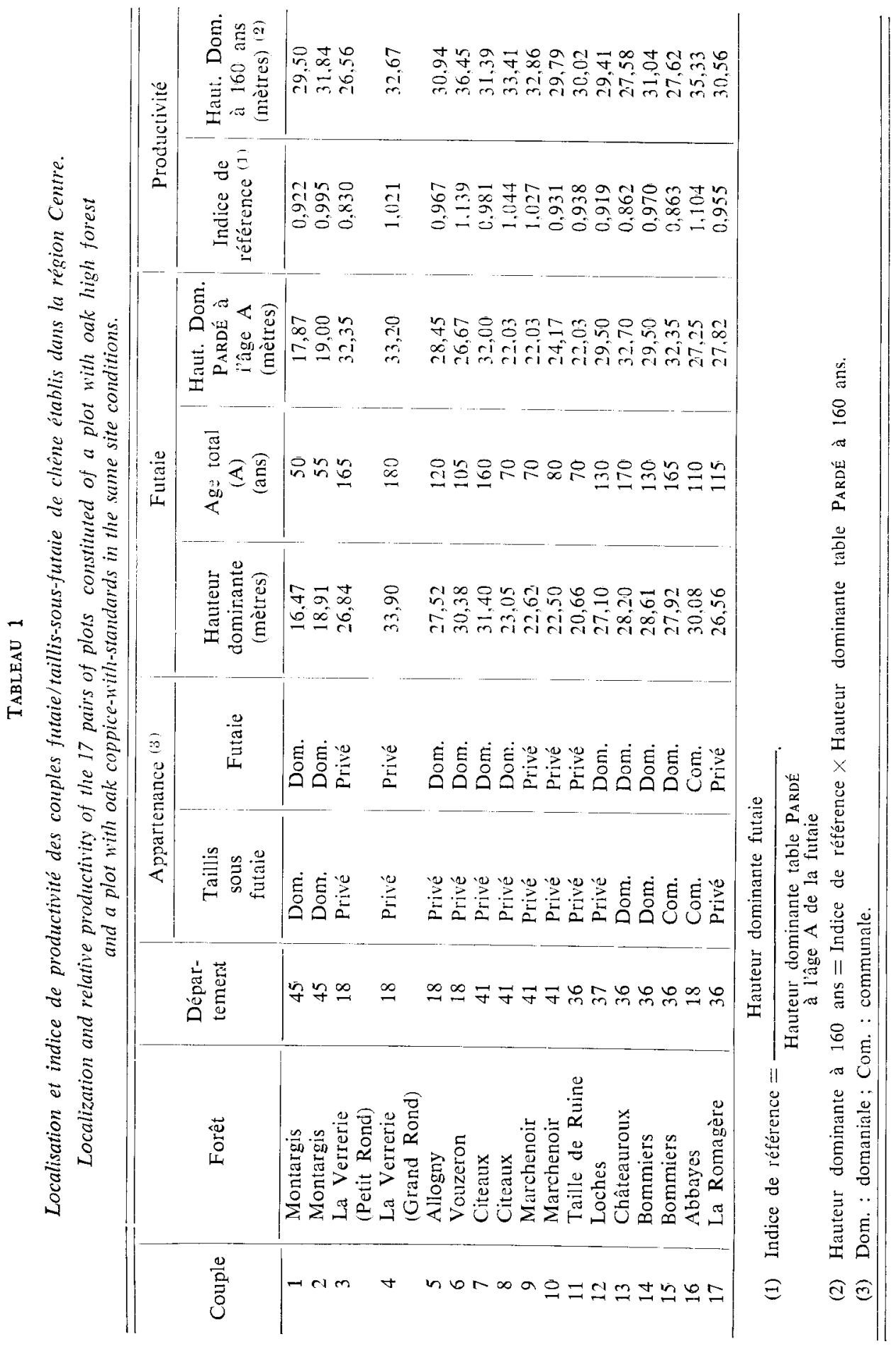




\subsection{Indice de productivité de référence}

\subsection{Principe de sa mesure}

La productivité relative des stations échantillonnées (nous ne les décrirons pas ici) peut être mesurée grâce à l'indice de productivité établi pour la futaie par référence à la table de production déjà citée de PARdé (1962), établie à partir de placettes permanentes installées sur sols de fertilité comparable et décrivant la croissance et la production moyenne de futaies de chêne rouvre ${ }^{131}$ de "bonne productivité (classe unique). La hauteur dominante d'un peuplement atteinte à un âge de référence a depuis longtemps prouvé son efficacité comme mesure de la productivité relative ; les conditions optimales de son estimation à partir d'études de croissance en hauteur basées sur des analyses de tiges darbres ont été exposées par ailleurs (LE GoFf, 1982). La méthode utilisée ici relève plus de celle à laquelle on doit recourir lorsque lon ne dispose que de mesures instantanées et qui consiste à déduire la croissance en hauteur pour différentes productivités d'une courbe moyenne établie à partir des données sur l'âge et la hauteur des peuplements mesurés, grâce à une transformation affine (7) : l'indice de productivité apparaît alors directement comme le rapport de la hauteur atteinte par un peuplement à un âge donné à la hauteur au même âge déduite de la courbe moyenne. La courbe de croissance en hauteur de référence utilisée ici est celle déduite de la table de production de Pardé et qui provient dun lissage par voie graphique des données des placettes permanentes précitées. L’indice de productivité des placettes de futaie de chêne, dénommé $\mathrm{I}_{\mathrm{F}}$ par la suite, a alors été évalué comme le rapport de lcur hauteur dominante à celle lue dans la table de PARDÉ au même âge (ou évaluée par interpolation entre deux âges consécutifs de la table). Les biais éventuels de la méthode de base (8) tiennent beaucoup à rétablissement de la courbe moyenne, et ne devraient done pas se retrouver ici tandis que le principe de courbes affines pour rendre compte de la croissance en hauteur, bien que souvent démenti, peut être acceptable dans un intervalle d'âges pas trop grand.

\subsection{Données recueillies et calcul de l'indice des couples}

I a hauteur dominante des placettes de futaie de chaque couple, définie comme la hauteur de l'arbre de surface terrière égale à la surface terrière moyenne des 100 plus gros arbres à l'hectare, a été déterminée après inventaire complet des circonférences des arbres (mesurées à $1,30 \mathrm{~m}$ ) dans des placettes circulaires de 20 ares (sauf pour 4 placettes (') $)$ et établissement d'une relation liant la circonférence et la hauteur des

(6) Ial distinction entre chêne rouvre et chêne pédonculé n’a pas ćć faite dans la présente étude : les chênaies de la région Centre son mixtes avec dominante du chêne pédonculé ou du chêne rouvre (pour les forêts de cette étude) suivant la nature du sol fd'apres la carte de la végétation potentielle de la France établic par Timbal (voir Becker et al., 1981)l.

(7) La transformation affine est telle que le rapport des hauteurs de deux de ces coubes à un âge donné est indépendant de l'âge.

(8) Des rappels plus détaillés sur cette méthode sont donnés par Orrorini et al. (1981, pp. 227-228), ainsi qu une amćlioration possible de celle-ci lorsque 1'on peut mettre en évidence leffet sur la croissance en hauteur d'un ou plusieurs facteurs du milieu (cas de laltitude pour le pin sylvestre en Margeride).

(9) Deux placettes de 15 ares, tune de 25 ares et unc de surface non délimitée (couple 13) en coupe de régénération où la hatuteur moyenne de 14 arbres restés sur pied a été prise comme estimation de la hauteur dominante. 
arbres d'un échantillon représentatif des classes de circonférence. L'âge de la futaie a été défini comme le nombre de cernes moyen compté sur des carottes de sondage effectuées à $30 \mathrm{~cm}$ de haut sur deux ou trois arbres et augmenté de 3 années pour tenir compte du temps mis pour atteindre cette hauteur. Les données hauteur dominante/âge de chacun des 17 couples ont été reportées sur la figure 1 qui présente également l'évolution de la hauteur avec l'âge pour les peuplements de futaie de la table de PARDÉ.

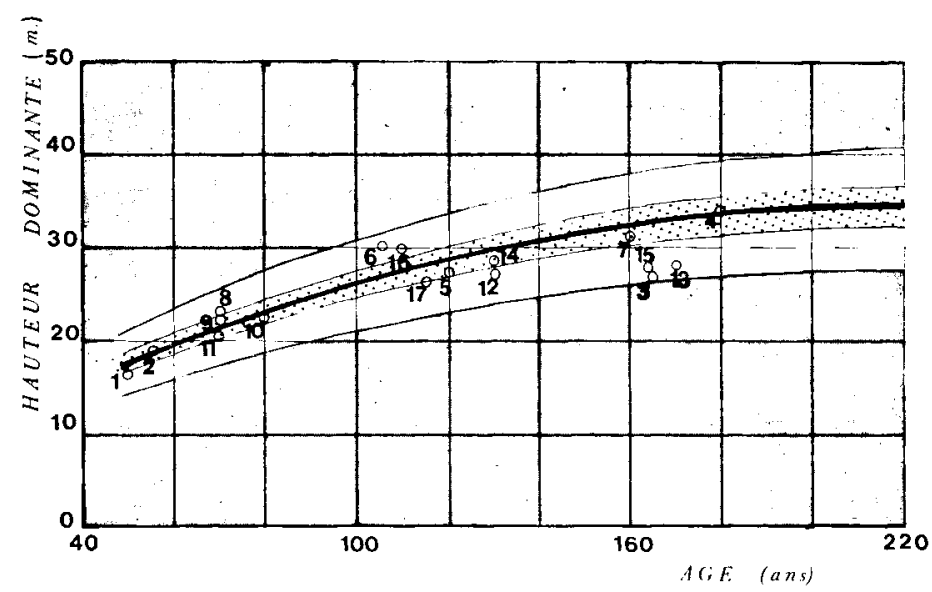

FiG. 1

Hauteur dominante et age des placettes de futaie des 17 couples futaie! taillis-sous-futaie.

Courbe de croissance en hauteur issule de la table de production de PARDÉ (trait plein) et courbes déduites par affinité (trait fin) délimitant trois classes de productivité pour les couples.

Top height and age of the oak high forest in the 17 experimental areas constituted of a pair of adjacent plots (high forest/coppice-with-standards) identical with regard to fertility.

Also shown are the top height/age curve for the single fertility class of the yield table for oak high forests in the Center of France (thick line) and derived fertility classes (delimited by thin lines).

Les indices de productivité de référence de chaque couple, déterminés selon le principe défini précédemment, figurent au tableau 1, de même que l'estimation qui en est déduite de la hauteur atteinte à l'âge de référence de 160 ans et qui permet de mieux juger de la gamme des productivités représentée : 8 couples sur les 17 se situent au niveau de la classe de productivité définie par la table, l'amplitude de variation de la hauteur dominante à 160 ans pour une classe étant fixée à 4 mètres ; 6 couples se situent au-dessous de ce niveau et seulement 2 au-dessus (fig. 2). Ainsi peut-on peut-être déjà situer le niveau de productivité équivalent au-dessus duquel un taillissous-futaie de chêne pcut être sans problème converti ou transformé, indice égal ou supérieur au minimum de la classe de PARdé par exemple, soit 30 mètres de hauteur dominante à 160 ans pour la futaie. 


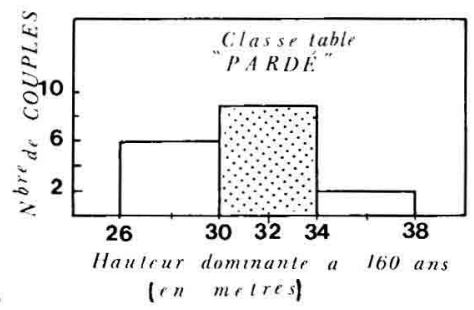

FIG. 2

Répartition des couples futaie/taillis-sous-futaie par classe de hauteur dominante de la futaie à 160 ans

(la classe 30 à $34 \mathrm{~m}$ correspond à celle de la table de production de PARDÉ).

Distribution of pairs of plots (high forest/coppice-with-standards) by classes of top height of oak high forest reached at age 160.

\section{Principes pour la recherche d'un indice de productivité des taillis-sous-futaie Recueil des données}

\subsection{Productivité et accroissement en circonférence des chênes de réserve}

On doit attribuer sans aucun doute au travail continu de générations de sylviculteurs ayant opéré dans les taillis-sous-futaie et contrôlé inventaire après inventaire (chacun séparé par une période de 20 à 30 ans, durée de révolution du taillis) l'évolution du matériel restant sur pied, d'avoir permis d'établir que «l'accroissement moyen en circonférence des réserves d'un taillis-sous-futaie, mesuré à périodicité fixe, est toujours figuré par une courbe sensiblement rectiligne, à partir de l'état de baliveau ou de jeune moderne et jusqu'aux plus fortes dimensions atteintes par l'espèce dans la station considérée» (Perrin, 1939). Il suffirait alors comme le dit De Lemps (1951), de prendre comme indice de productivité une échelle d'accroissement moyen en circonférence des réserves et pour plus de facilité même, de ne considérer que les arbres parvenus au terme d'exploitabilité du peuplement et dont il est facile de retrouver l'âge au moment de la coupe. DE LEMPS a pu ainsi caractériser la productivité de quatre types de stations (définis auparavant pédologiquement) de la région de Dijon dont l'accroissement moyen en circonférence des arbres réservés âgés de 125 à 150 ans variait de 30 à $60 \mathrm{~cm}$ environ par révolution de 25 ans.

L'utilisation effective de cet indice, tout trouvé, pour les taillis-sous-futaie de la région Centre ne serait cependant envisageable que pour ceux dentre eux conduits «normalement», les coupes de taillis et d'éclaircie ayant été faites de façon régulière, la composition en réserves comparable, le terme d'exploitabilité identique ou proche : en somme des conditions de peuplement bien définies; nul doute en effet qu'elles conditionnent l'indépendance de l'accroissement moyen en circonférence des réserves vis-à-vis de facteurs autres que la fertilité de la station. La condition des taillis-sous-futaie actuels en forêt privée est toute autre : pour beaucoup, les coupes régulières et les interventions culturales qui y sont liées ont été interrompues, pour d'autres une surexploitation des réserves a été opérée, entraînant en particulier une raréfaction des vieux arbres; l'inventaire des placettes de taillis-sous-futaie de chaque couple donnera un aperçu de ces conditions diverses actuelles (voir le paragraphe suivant et le tableau 2). 
I1 n’en reste pas moins que l'accroissement en circonférence (à $1,30 \mathrm{~m}$ de haut) des réserves du taillis-sous-futaie est directement lié à la fertilité ; la considération des autres facteurs ayant pu influer sur cet accroissement et leur prise en compte a finalement orienté la méthodologie proposéc ainsi que l'échantillonnage et le recueil des données pour cette étude.

L'accroissement en circonférence est la différence entre les circonférences mesurées à deux moments donnés; cet accroissement étant par ailleurs difficile à estimer sans observations suivies, ciest la croissance en circonférence reconstituée à partir d'un ensemble de données instantanées recueillies sur des arbres d'âges variés, qui constituera la base de cette étude : le parallèle peut être fait facilement avec la méthode utilisée pour reconstituer la croissance en hauteur avec des données de même nature (voir $2.21)$ et en déduire les courbes d'évolution moyenne pour des niveaux de productivité donnés. Les résultats acquis antérieurement, et dont il vient d'être fait état, sur l'accroissement des chênes de réserve, notamment ceux de PERRIN (1939), seront cependant d'une grande utilité pour la recherche de la forme de la relation liant la circonférence à lâge et aux autres facteurs pris en considération : vigueur ${ }^{101}$ des arbres liée au potentiel génétique), concurrence subie antérieurement (de la part du taillis et d'autres réserves éventucllement) et mesurée par une variable pouvant rendre compte globalement cie ces effets, la hauteur relative du houppier (rapport de la hautcur du houppier à la hauteur totale de larbre) '"', fertilité de la station enfin dont on cherche la mesure.

Le graphique de la figure 3 reproduisant les résultats de PERrin suggère ainsi que, pour des conditions de concurrence identiques (traitement moyen sembiable), les relations liant la circonférence à l'âge pour différents niveaux de productivité et pour des arbres de vigueur différente (arbres d'élite, moyens, retardataires), sont des c'roites qui sc déduisent les unes des autres sensiblement par affinité.

Enfin, si rien n’a été dit sur la possibilité, comme cela est plus habituel. de se baser plutôt sur la croissance en hauteur pour un tel genre détude, c'est que l'on ¿ispose d'informations moins précises sur lallure de la croissance en hauteur, dont on sait par ailleurs qu'elle est influencée par le traitement en taillis-sous-futaie (12) (voir BAR'TET, 1891). De même, le recours à la hauteur des plus vieilles réserves comme indice de productivité (la croissance en hauteur serait très réduite après 150 ans), utilisé avec un relatif succès dans certaines études (BECKER, 1979), ne pouvait être ici envisagé pour les raisons édictées plus haut et relatives à la composition actuelie des taillis-sous-futaie, en partici:er le manque fréquent de vie::!es réserves.

(10) Dans cette étude, Ja vigueur d'un arbre dâge donné traduira ses performances de croissance en circonférence, toutes conditions extérieures à l'arbre étant égales par ailleurs (fertilité de la station, statut concurrenticl). Dans une population darbres d’àe donné, on pouma ainsi définir des classes de vigueur pour les arbres: selon, par exemple, que leur circonférence est supérieurc. égale ou inféricure à la valeur médiane des circonférences obscrvées dans la population : pour les premiers cités, on parlera alors darbres d'élite et c'est à eux que lon s'intéressera surtout dans ce travail.

(11) Les propriétés de la hauteur relative du houppier en tant que mesure de densité ont pu être déjà utiïisées (LE, GOFF \& OTlORINI, 1979), mais son utilisation la pius concrète apparait dians un article de CURTIN (1970).

(12) Cette influcnce a pu êtrc confirméc indirectement dans cette étude et des recherches sont poursuivies, dont il scra fait étal par ailleurs, pour tenter de rendre compte plus précisément de l'effet réel du traitement en taillis-sous-futaie sur la croissance en hauteur. 


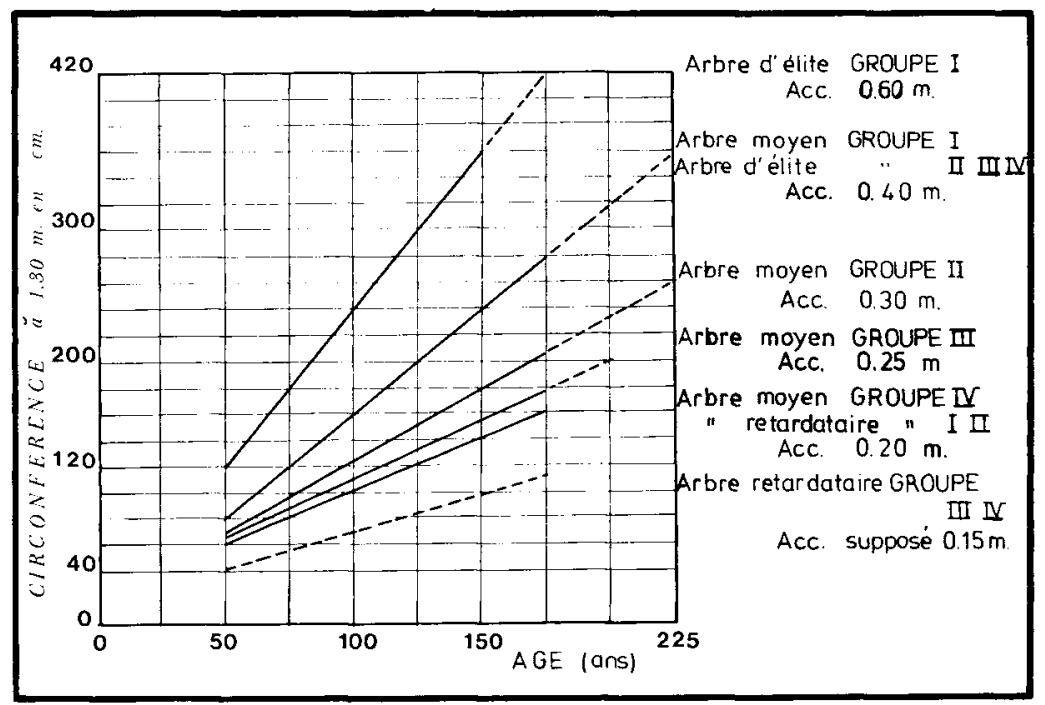

FIG. 3

Développement en circonférence des chênes de réserve des taillis-sous-futaie dhe Bassin de la Saône pour 4 groupes de stations (d'après PERrin, 1939).

«Acc» désigne l'accroissement sur lá circonférence pour une période de 25 ans.

Groupe 1 : Sols d'alluvions modernes fraîches ct riches.

Groupc II : Sols marneux ou argileux de qualité moyenne.

Groupe III : Sols calcaires marneux, généralement méliocres.

Groupe IV : Sols de calcaires compacts, extrêmement superficiels.

Breast height girth development of the standards in coppice-with-standards of the "Bassin de la Saone» for 4 soil types.

(«Acc» is for girth increase during a 25 years period).

\subsection{Recueil des données}

En taillis-sous-futaie conduit normalement, c'est-à-dire suivant une norme fixant le nombre d'arbres à laisser dans chaque classe d'âge, la distribution des arbres en classes de circonférence se présente sous la forme d’une série de courbes «en cloche» chacune relative à une classe d'âge du taillis-sous-futaie. Dans ce cas, l'âge d'un arbre quelconque peut théoriquement s'évaluer assez précisément si l'on connaît l'âge des plus vieilles réserves (comptage des cernes sur leurs souches une fo:s abatius) ou celui de la dernière coupe, en supposant toutefois que les coupes soient intervenues régulièrement. Rien de tel lorsque la structure normale n'existe plus, ce qui doit être le cas pour la majorité des taillis-sous-futaie de la région concernés par cette étude et dont le traitement normal n'est plus suivi ; c'est le cas également pour les taillis-sousfutaie en voie de conversion quiil a été nécessaire de retenir pour disposer d'un nombre suffisant de couples.

Aussi, à cause de la nćcessité de sonder à la tarière les arbres échantillonnés pour connaître leur âge, l'échantillonnage a-t-il été limité aux arbres d'élite normalement situés dans la partie droite de l'histogramme des circonférences relatif à chaque classe 
d’âge ; leur choix n'a pas été toujours conforme aux prévisions (cf. fig. 4), mais l'échantillonnage réalisé permettra cependant la sélection d'un certain nombre d'arbres parmi les plus vigoureux du taillis-sous-futaie sur lesquels sera baséc la mesure de la productivité, retrouvant par-là l'idée de DE LFMPs étendue, si l'on peut dire, à toutes les classes d'âge.

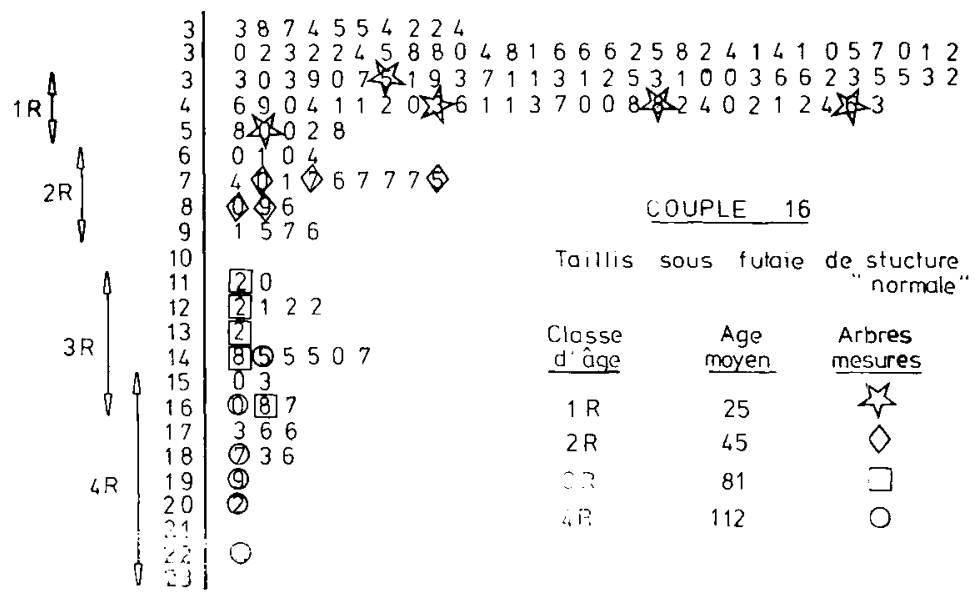

FiG. 4

Relevé (1) des valeurs de circonférences (prises à $1,30 \mathrm{~m}$ de haut)

faisant apparaître une distribution en classes de $10 \mathrm{~cm}$, chaque mesure étant représentée par le nombre de centimètres dépassant la valeur la plus basse de la classe (la classe de $30 \mathrm{~cm}$ a été démultiplicée pour la représentation).

Exemple : $11: 20$ correspond à 2 mesures de 112 et $110 \mathrm{~cm}$ dc circonférence.

Statement of breast height girth values measured for a coppice-with-standards plot (pair n" 16$)$; girth classes are $10 \mathrm{~cm}$ large and the girth value for a tree is represented by the number of centimeters above the lower limit of the required class

(see Mosteller \& Tukey, 1977, pp. 43-48 for details on this type of data statement).

Cette figure, comparable à un histogramme, fait directement apparaître 4 classes d'âge notées $1 R, 2 R, 3 R, 4 R$, où $R$ désigne la période de rotation des coupes (ou durée de révolution du taillis); les arbres sondés et mesurés dans chaque classe (repérés par les différents symboles du tableau ci-dessus), montrent le recouvrement des classes $3 \mathrm{R}$ et $4 \mathrm{R}$ et explique ainsi la présence dans l'échantillon, d’arbres de circonférence inférieure à la valeur médiane des classes : ex. le 145, le 160.

This girth values statement allow's to recognize age classes of the coppice-with-standards stands $(4$ age classes here called $1 R, 2 R, 3 R, 4 R ; R$ is for coppice revolution period). Trees sampled are identified by the above table symbols.

(i) Ce type de relevé est décrit plus complètement dans Mosteilnis \& Tukey (1977, pp. 43-48). 


\section{- Inventaire}

Afin d’avoir une bonne représentation des différentes classes d'âge (le nombre d"arbres à l'hectare dans chaque classe décroissant fortement avec l'âge), des placettes de surface généralement comprise entre 0,5 et 1 hectare et ayant la forme d'un quadrilatère, ont été délimitées sur le terrain; la nécessité d’avoir des conditions de station et de peuplement homogènes a cependant conduit à limiter la surface de certaines d'entre elles. Le tableau ci-dessous en donne la répartition par classes de surface :

\begin{tabular}{|c|c|c|c|c|c|c|}
\hline \multirow[b]{2}{*}{ Placettes } & \multicolumn{6}{|c|}{ Surface des placettes de taillis-sous-futaie (ares) } \\
\hline & $30-40$ & $40-50$ & $50-60$ & $60-70$ & $70-80$ & $80-90$ \\
\hline Effectif & 4 & 1 & 5 & 5 & 1 & 1 \\
\hline Couples & $11,13,14$ & 1 & $\begin{array}{c}6,9,12 \\
16,17\end{array}$ & $2,5,7,8$ & 4 & 3 \\
\hline
\end{tabular}

La circonférence à $1,30 \mathrm{~m}$ de tous les arbres a été mesurée et les valcurs supérieures à $20 \mathrm{~cm}$, seules retenues, ont été reportées directement sur imprimé de la façon décrite à la figure 4 ; ce mode de report a lavantage de faire apparaître directcment la forme de la distribution et de permettre de conserver les valeurs exactes mesurées.

\section{TABLEAU 2.}

Résultats de l'inventaire des placettes de taillis-sous-futaie de chaque couple donnant la répartition des arbres en classes de circonférence.

Results from the inventory of the coppice-with-standards plots giving the distribution of trees in breast height girth classes (girth classes are intimately linked to age classes).

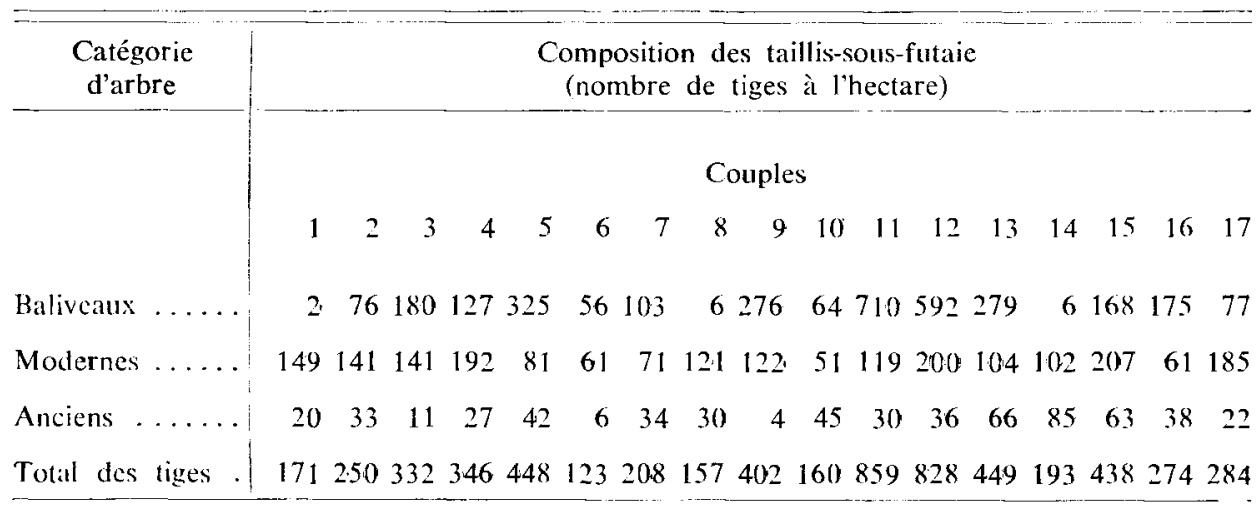

(Baliveaux : 10 à $50 \mathrm{~cm}$; Modernes : 50 à $130 \mathrm{~cm}$; Anciens : 130 à $270 \mathrm{~cm}$; ces trois classes de circonférences correspondent grossièrement à des arbres dont l'âge va croissant.) 
Les résultats condensés des inventaires (tabl. 2) font apparaître des disparités importantes dans la composition des taillis-sous-futaie des différents couples, illustrant ce qui a été dit précédenment et conséquence de l'éloignement du traitement normal en tailits-sous-futaie pour certains peuplements. Bien quiil ait été tenté de pousser plus loin lanalyse de la structure de chaque peuplement de taillis-sous-futaic, notamment par comparaison avec les normes théoriques établies par De Lemps (1951), il n’en sera pas fait état ici ; les conséquences des différences de traitement apparaîtront cependant clairement à travers l'évaluation du statut concurrenticl des arbres échantillonnés qui, nous le verrons au paragraphe suivant, permettra dexpliquer une partie de la variabilité des c:rconférences observées à un âge donné.

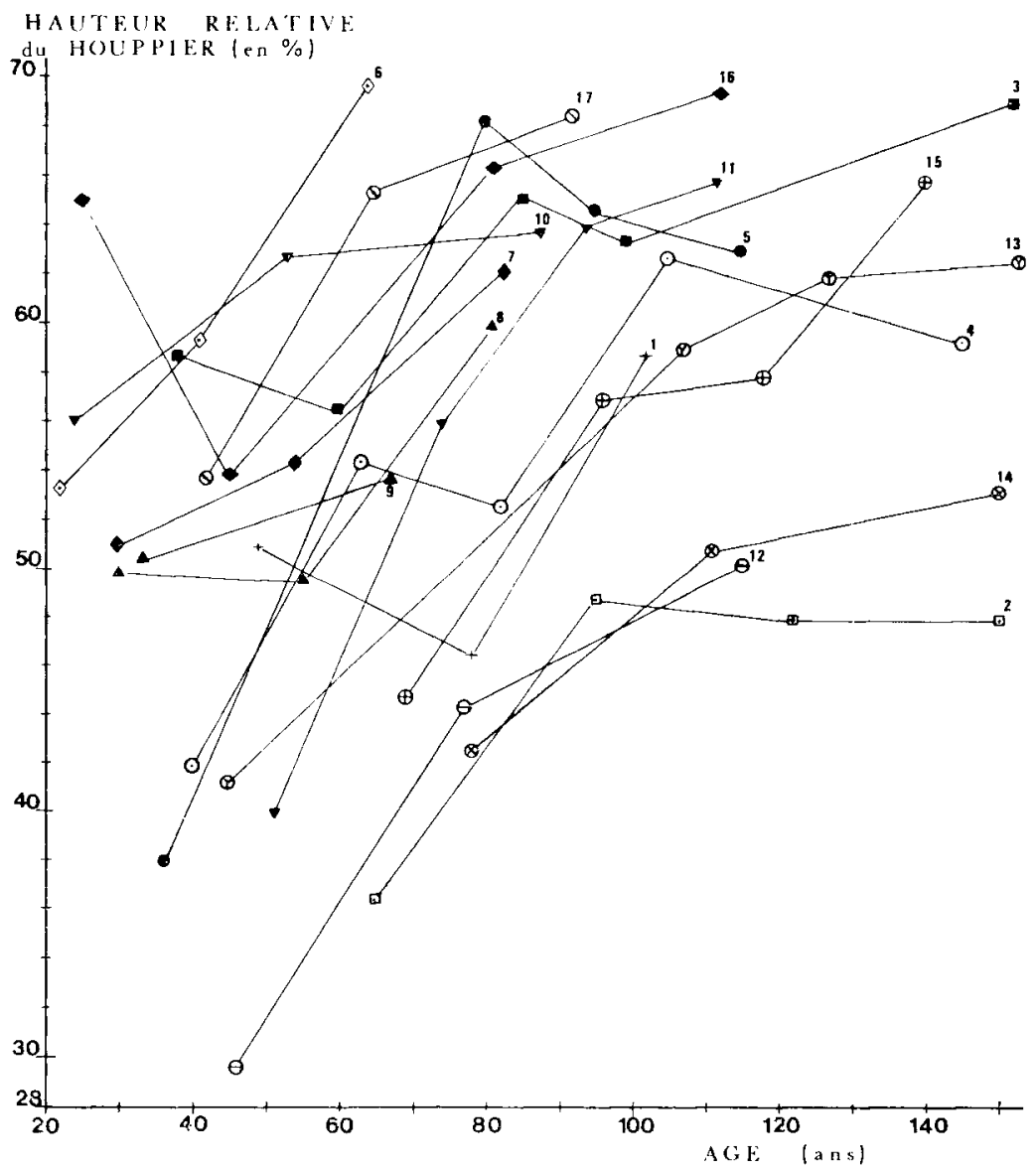

Fig. 5

Hauteurs relatives movennes du houppier des arbres échantillonnes dans les différentes classés d'àge du taillis-sous-futaie de chaque couple.

Mean relative heights of crowns of sampled trees for the different ase classe's of the coppice-with-standards experimental plots. 
- Echantillonnage et mesures

Un échantillon de 5 arbres choisis parmi les arbres d'élite, dans chaque classe d'âge repérée sur l'histogramme des circonférences des placettes de taillis-sous-futaie (cf. figure 4), a fait l'objet des mesures suivantes, outre celle de leur circonférence à $1,30 \mathrm{~m}$ (mesure au ruban) : hauteur totale et hauteur du houppier (la base du houppier étant définie par le niveau de la première branche participant effectivement au houppier de l'arbre), mesures effectuées au Blume-Leiss, âge après comptage des cernes annuels sur des carottes de sondage (1\%).

A partir de ces mesures, l'évaluation du statut concurrentiel de chaque arbre mesuré par la hauteur relative du houppier (cf. 3.1.) a été effectuée. Les valeurs moyennes (exprimées en pourcentage) pour les arbres d'une même classe d'âge ont été calculées dans chacun des couples; ces valeurs sont reportées à la figure 5 en fonction de l'âge moyen des arbres. Il est ainsi possible de se rendre compte de la variabilité importante des conditions de densité rencontrées dans ces taillis-sous-futaie : la hauteur moyenne du houppier des arbres est comprise entre 30 et 70 p. 100 de la hauteur totale. Il faut noter cependant que des valeurs inférieures à 45 p. 100 ne se rencontrent que pour des âges inférieurs à 100 ans. A l'intérieur de chaque couple, la hauteur relative du houppier a tendance à augmenter avec l'âge, traduisant des conditions de concurrence plus fortes dans le jeune âge. Cependant, globalement, la diversité des statuts concurrentiels pour les arbres se retrouve aux différents âges, mis à part pour les plus fortes densités comme signalé ci-dessus; ces faits apparaissent clairement dans le tableau qui suit :

\begin{tabular}{c|c|c|c|c|c|c|c}
\hline $\begin{array}{c}\text { Hauteur relative } \\
\text { du houppier } \\
\text { (cn \%) }\end{array}$ & $0-25$ & $25-50$ & $50-75$ & $75-100$ & $100-125$ & $125-150$ & $150-175$ \\
\hline Minimum ..... & 40 & 17 & 23 & 30 & 32 & 49 & 48 \\
Maxinum .... & 78 & 74 & 77 & 77 & 79 & 75 & 76 \\
\hline
\end{tabular}

La mise en évidence de leffet de la concurrence, en fait des conditions de concurrence passées (synthétisées de façon simplifiée par la hauteur relative du houppier observée), sur la croissance en circonférence des arbres ainsi que sa prise en compte dans la relation de prédiction de la circonférence, fait lobjet du paragraphe suivant; la réduction ainsi obtenue de la variabilité non expliquée de la circonférence atteinte à un âge donné, permettra dans la dernière phase de létude de proposer une meilleure mesure de la productivité relative des taillis-sous-futaie.

\section{Analyse et traitement des données}

Nous rappelons ici que les facteurs attendus pour expliquer les variations observées de la circonférence des arbres sont laage, les conditions de concurrence passées, la

(13) Les sondages ont été effectués à $1,30 \mathrm{~m}$ de haut et, pour avoir làge total, on a ajouté al nombre de cernes lu, le nombre d'années mis pour atteindre $1,30 \mathrm{~m}$ évalué sur les arbres les plus jeunes par un sondage supplémentaire au pied. 
vigueur et enfin la fertilité de la station. Le présent paragraphe aura pour objet de mettre en évidence l'effet des deux premiers facteurs. les variations de la vigueur des arbres étant considérées comme aléatoires (il s`agit normalement seulement darbres d'élite), celles dues à la fertilité devant être appréciées en fonction de l'écart à la relation moyenne qui sera ainsi établic. Ainsi, c'est la dépendance de la circonférence à $1,30 \mathrm{~m}$ vis-à-vis de l'âge et de la hauteur relative du houppier, qui sera étudiée ici.

L'étude de cette dépendance s’appuiera sur la connaissance de certains faits (cf. 3.1.) et la formulation d'hypothèses, lanalyse des données proprement dite, venant seulement confirmer et préciser lallure des phénomènes. La méthode utilisée pour l'analyse des données est celle exposée par OTTORINı (1981, pp. 489-490) : elle consiste à observer par voie graphique la dépendance de la variable à expliquer vis-à-vis d'une des variables explicatives, à ajuster une relation provisoire une fois la forme de dépendance reconnue (éventuellement après transformation de variable pour se ramener à une forme linéaire) et à examiner ensuite, avec les mêmes techniques, la dépendance des valcurs résiduelles provenant de lestimation obtenue avec la relation provisoire vis-à-vis des autres variables explicatives retenues.

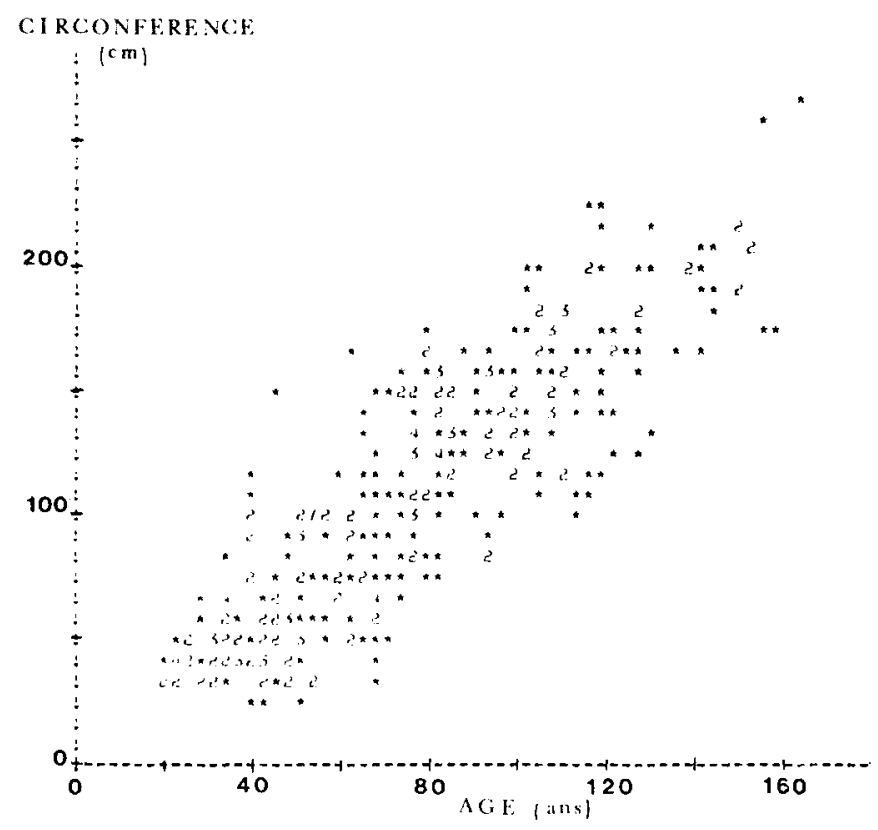

Frg. 6

Age et circonférence des réserves de taillis-sons-funaie :

l'échantillon est constitué par des arbres choisis parmi les plus vigoureux dans les différentes classes d'áge représentées dans chaque peuplement (voir 3.2.).

Scatter plot of the sampled coppice-with-standards trees girth (measured at breast height) against age: trees sampled (= picked trees) are chosen in each "presumed $\$$ age class of the coppice-with-standards plots so that their girth is superior or equal to the median girth of the class (se'e also fig. 4). 
La forme reconnue de dépendance de la circonférence vis-à-vis de l'âge pour les réserves de taillis-sous-futaie est celle d'une relation linéaire (cf. fig. 3). La figure 6 présente les couples de valeurs de la circonférence et de l'âge pour les 344 arbres qui ont été mesurés dans les 17 couples : la dépendance linéaire se trouve confirmée, même s'il semble que l'on observe une légère courbure qui peut être due à l'échantillonnage (les arbres les plus âgés appartiennent en moyenne à des couples sur stations moins fertiles : 3 des 6 couples représentés $(15,13,3)$ se situent dans la moins bonne classe de fertilité (cf. fig. 1). Une relation provisoire de la forme $\mathrm{C} 130=\mathrm{a}+\mathrm{b}$ Age a ainsi été ajustée à ces données.

La dépendance de C 130 vis-à-vis de la hauteur relative du houppier (HC/HT) peut être mise en évidence en partageant le nuage de points de la figure 1 suivant plusieurs classes d'âge et en calculant pour chacune d'elles les valeurs moyennes observées de la circonférence pour différentes classes de $\mathrm{HC} / \mathrm{HT}$. La figure 7 présente les résultats obtenus alors en portant en abscisse la valeur moyenne observéc de $\mathrm{HC} / \mathrm{HT}$ dans chacune des classes constituées $(15$ à 30 p. $100 ; 30$ à 45 p. $100 ; 45$ à 60 p. 100 ; 60 p. 100 et plus) et pour chaque classe d'âge. On peut ainsi observer que la circonférence moyenne croît avec $\mathrm{HC} / \mathrm{HT}$ (si l'on exclut les points correspondant à des classes peu représentées) et ce dautant plus que l'âge est élevé : la pente des segments de droite reliant des points pris dans deux classes de $\mathrm{HC} / \mathrm{HT}$ mitoyennes croît avec l'âge, excepté pour les classes lâge les plus élevées pour lesquelles on peut faire la même remarque quà l'alinéa précédent.

L'interaction entre deux variables indépendantes peut être observée directement en analyse de données en reliant la différence des logarithmes de la grandeur observée et de son estimation provisoire, avec la variable dont on veut tester linteraction (14) (Mosteller, 1977, p. 435) : l'interaction entre lâge et la hauteur relative du houppier a pu ainsi être confirmée (fig. 8a) et la forme sous laquelle intervient $\mathrm{HC} / \mathrm{HT}$ précisée, après transformation logarithmique de cette variable (fig. 8b). Les valeurs résiduelles obtenues après ajustement linéaire de C 130 en fonction de la nouvelle variable Age $\times \log (\mathrm{HC} / \mathrm{HT})$, portées en fonction des variables originelles Age et $\mathrm{HC} / \mathrm{HT}$, ont montré seulement un effet linéaire complémentaire de $\mathrm{HC} / \mathrm{HT}$ exprimé sous la forme de son logarithme.

A ce stade, il restait alors à estimer les paramètres de la relation suivante liant la circonférence à l'âge et à la hauteur relative du houppier :

$$
\mathrm{C} 130=\mathrm{a}+\mathrm{b} \log \mathrm{HC} / \mathrm{HT}+\mathrm{c}(\mathrm{Age} \times \log \mathrm{HC} / \mathrm{HT})
$$

La relation (1) a été ajustée par la méthode habituelle des moindres carrés utilisée en régression linéaire. L'examen simultané des valeurs résiduelles et des valcurs estimées, recommandé après un ajustement (DRAPER \& SMITH, 1966), a montré une augmentation de la dispersion des valeurs résiduelles assez nette dans l'intervalle des circonférences estimées compris entre 20 et $100 \mathrm{~cm}$; une surestimation des faibles valeurs de la circonférence a pu aussi être observée. Afin de corriger cet effet, une méthode dérivée de celle des moindres carrés, dans laquelle on fait intervenir des pondérations a été employée (15) (Mostfaler \& TukEy 1977, p. 356). Un examen des nouvelles valeurs

(14) Ceci n'est vrai cependant que si l'ajustement déjà réalisé est suffisamment bon.

(15) Pour plus de détails et une illustration de cette méthode d'estimation, on pourra se référer à OTtORini et al. (1981, pp. 229-231). 


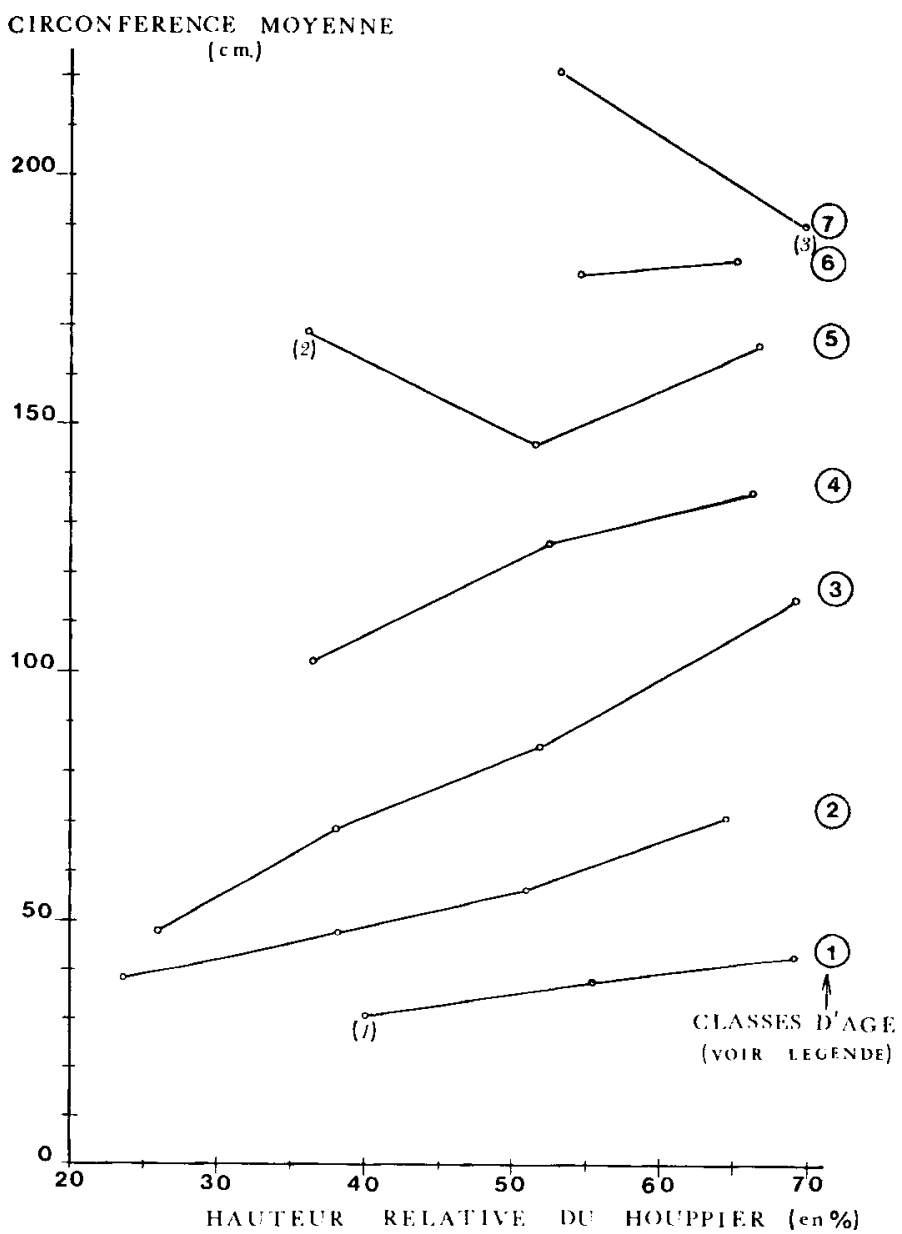

FIG. 7

Circonférence moyenne des réserves de taillis-sous-futaie échantillonnées, observée pour des valeurs croissantes de la hauteur relative du houppier

à l'intérieur de classes d'âge déterminées ( $I: 0-25$ ans, $2: 25-50$ ans, $3: 50-75$ ans, $4: 75-100$ ans, $5: 100-125$ ans, $6: 125-150$ ans, $7: 150-175$ ans).

Mean breast height girth of sampled coppice-with-standards trees of increasing relative heights and belonging to different age classes.

(On a fait ligurer entre parenthèses le nombre d'observations attachées à un point lorsque celui-ci était inféricur à 5 ).

(Bracketed numbers on this figure represent the number of trees for one observation when this number is less than 5). 


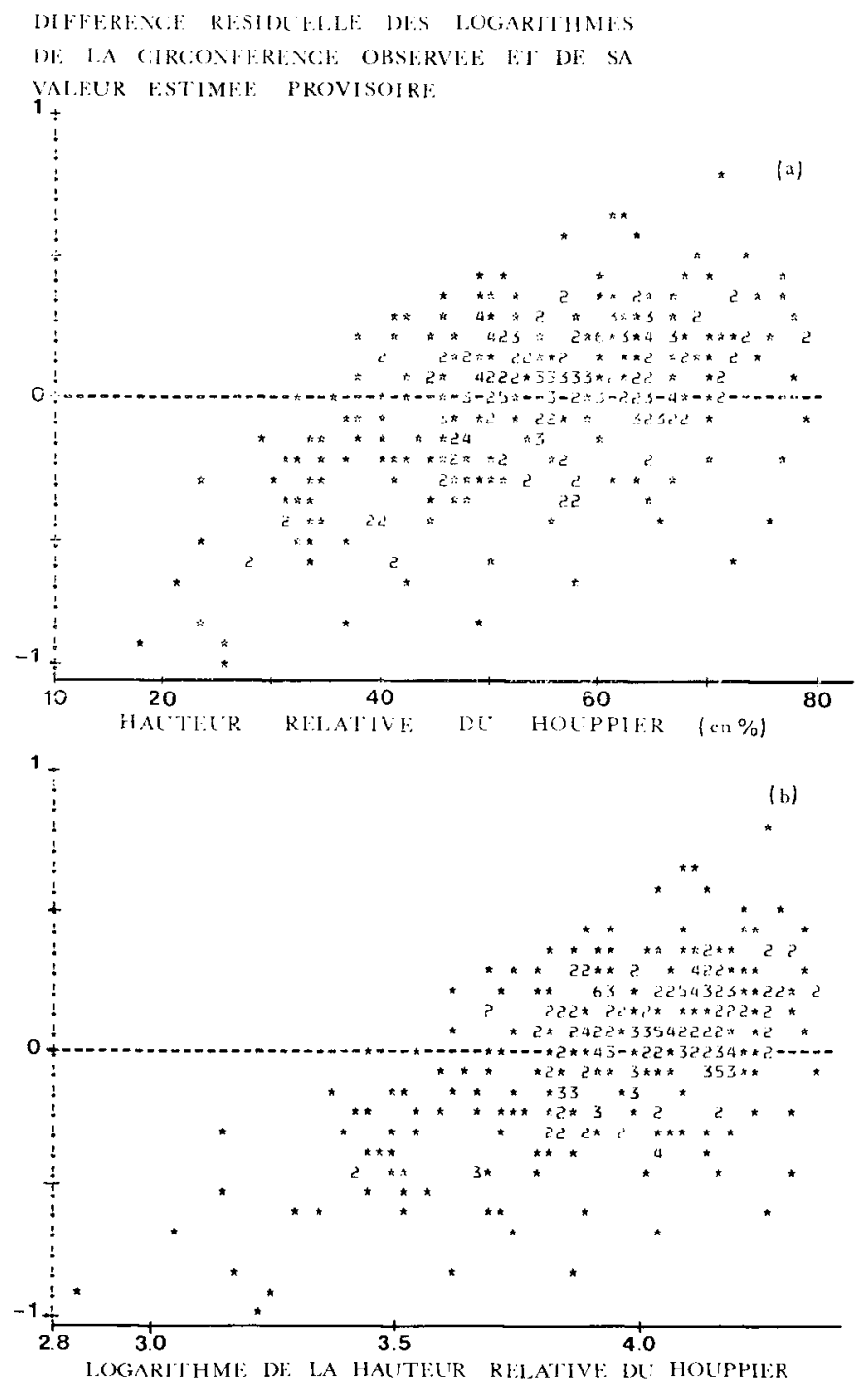

FiG. 8

Dispersion des valeurs de la différence résiduelle

entre les logarithmes des circonférencés observées ét des circonférences cotimées par la relation provisoire C $130=a+b$ Age:

(a) en fonction de la hateut relative du houppier,

(b) en fonction du logarithme de la hauteur relative du houppier.

La figure 8(a) permet de mettre en évidence l'interaction entre les variables indépendantes âge et hauteur relative du houppier; la figure $8(\mathrm{~b})$ révèle sous quelle forme intervient cettc nouvelle variable cxplicative (voir le texte pour plus de détails).

Scatter plot of the residual differences between the logarithms of the observed values of breast height girth of standard trees and those estimated by the provisional relationship established between girth and age : $C 130=a+b$ Agc

a) against the relative height of the crown of standard trees;

b) against the logarithm of the relative height of the crown of standard trees. 
résiduclles et des valcurs estimées ne montre pas cette fois-ci de surestimation ou de sous-estimation de la circonférence dans l'intervalle des valeurs estimées. Les paramètres de la relation (1) ont été obienus après plusjeurs ajustements suceessifs faisant intervenir des pondérations calculées de façon itérative : le processus a été arrêté lorsque les estimations obtenues ne se modifiaient pius que faiblement. Les valeurs finales estimées des paramètres de la relation (1) sont les suivantes :

$$
\begin{aligned}
& \hat{a}=-116.8 \\
& \hat{b}=32.6335 \\
& \hat{c}=0.313705
\end{aligned}
$$

avec les unités suivantes pour les grandeurs représentées : circonférence (C 130) en centimètres, âge en années, hauteur relative du houppier $(\mathrm{HC} / \mathrm{HT})$ en pourcentage.

La figure 9 est une illustration de la relation (1) : le comportement de la croissance en circonférence y est représenté pour différentes valeurs de la hauteur relative du houppier, dans le domaine des observations réalisées. L'interprétation suivante peut en être faite : pour des arbres gardant le même statut concurrentiel (valeur fixe de $\mathrm{HC} / \mathrm{HT}$ ), l'accroissement de la circonférence reste constant avec l’âge pour une période de temps donnée; il croît lorsque les arbres sont moins concurrencés proportionnellement au logarithme de la hauteur relative du houppier.

\section{Indice de productivité des taillis-sous-futaie de chêne}

A ce stade de l'étude et pour atteindre l'objectif fixé. il restait alors à tenir compte, en plus des résultats qui viennent d’être obtenus, de ceux établis par Plerrin en 1939 đont il a été fait état au paragraphe 3.1. ct illustrés par la figure 3. Si lindice I caractérise la fertilité de la station et lindice $v_{i}$ la vigueur d’un arbre $i$ sur cette station, la circonférence à $1,30 \mathrm{~m}$ de cet arbre à l'âge $\mathrm{A}$, à un facteur aléatoire près est donnée par :

$$
(\mathrm{C} 130)_{i}=\mathrm{f}\left[\mathrm{A}_{\mathrm{i}},(\mathrm{HC} / \mathrm{HT})_{i}\right] \mathrm{I} \cdot \mathrm{v}_{\mathrm{i}}
$$

où $\mathrm{f}$ est la fonction représentée dans la relation (1); on se trouve bien ainsi en accord avec les résultats de PERRIN : proportionnalité des courbes de croissance en circonférence lorsque la fertilité de la station varie et que change lo niveau de vigueur de l'arbre (cf. arbres d'élite, moyens, retardataires de la figure 3). Pour une placette de taillis-sous-futaie j, la relation (2) fournit :

$$
\left\{v_{i}\right\}_{j}=\left\{\frac{(\mathrm{C} 130)_{i}}{\mid f\left[A_{i},(H C / H T)_{i}\right]}\right\}
$$

où $(C .130)_{i}$ est la circonférence observée de l'arbre $i$ et $f\left[A_{i},(H C / H T)_{i}\right]$, sa circonférence estimée, soit $(\mathrm{C} \widehat{130})_{i}$, par la relation (1).

Si l'échantillon des arbres de chaque placette de taillis-sous-futaie était de même vigueur moyenne $\mathrm{V}$, la relation (3) appliquée à tous les arbres fournirait directement 


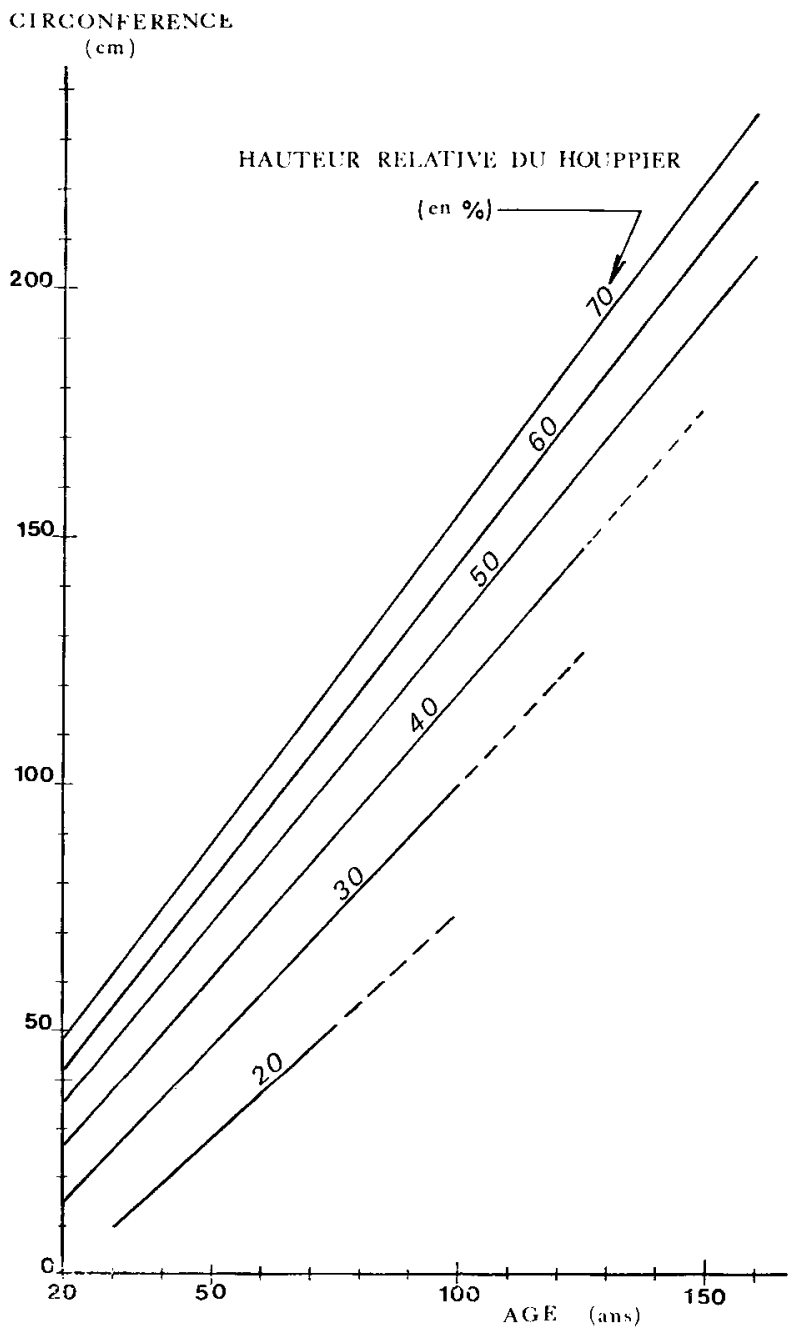

F1G. 9

Développement caractéristique de la circonférence des réserves de taillis-sous-futaie dans des conditions de concurrence variables

définies par la hauteur relative du houppier des arbres (variant ici de 20 à $70 \%$ ).

Characteristic development of the breast height girth of the standard trees in the coppice-with-standards with varied values of the competitive status of trees measured by the relative height of the crown of the standards (varying here from $20 \%$ to $70 \%$ ) 
C OLPLES

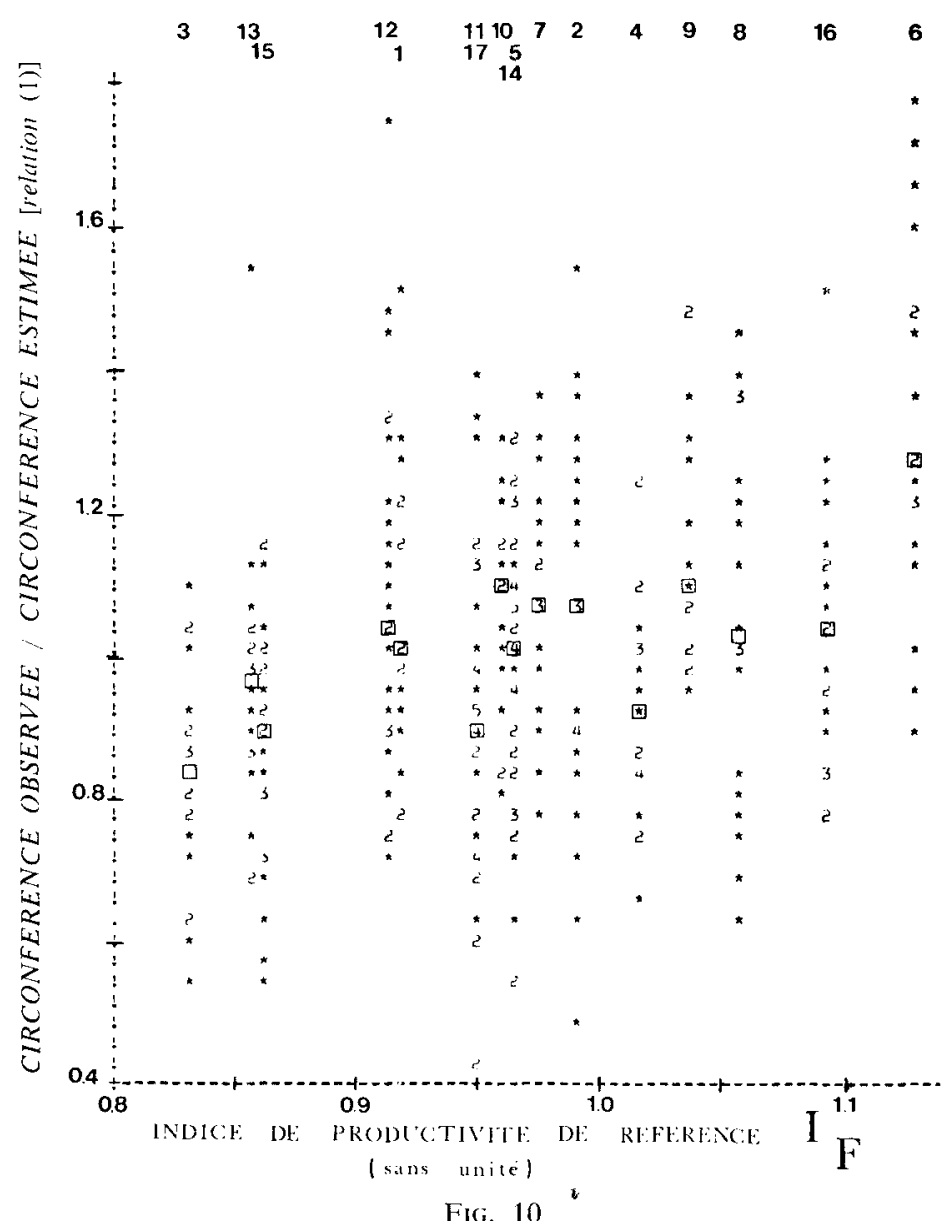

Dispersion des valeurs du rapport de la circonference observée à la circonfórence estimée Là laide de la relation (I)] des réserves de taillis-sotis-futaie, en fonction de l'indice de productivité $I_{\text {r }}$

basé sur la haiteur dominante du peuplement de futaie de chaque couple (voir 2.2).

Scatter diagram of the values of the ratio of observed breast height girlh of the standards to girth estimated with relation (I)

(in which girth is a function of both age and relative height of crown) against the relative productivity index $\left(I_{n}\right)$ of each pair of plots based on top height of the high forest.

Ce rapport apparaît lié linérairement à $\mathrm{I}_{\mathrm{F}^{\prime}}$ (les valeurs médiancs (1), pour des niveaux variables de $I_{b}$, se situent sur une (lroite) et il sert de base à la définition de l'indice de productivité pour les taillis-sous-futaic.

( $I_{F}$ is the ratio of the top height of the high forest to top height at the same age as determined for the single fertility class of the yield table for oak high forests in the Center of France : see (ig. 1).

(1) Elles sont représentécs par le symbole $\square$ 
un indice de productivité, soit I.V. (16), l'indice proprement dit I étant multiplié par la constante V. Il a été signalé cependant précédemment (voir 3.2.) que l'échantillonnage réalisé n'a pas toujours permis cé sassurer đune même classe de vigueur pour tous les arbres d'une même placette, en particulier pour celles dont la structure différait de celle d'un taillis-sous-futaie normal; les différences de vigueur moyenne des arbres d'une placette à lautre apparaissent à la figure 10 où ont été reportées les valeurs de C $130 / \widehat{C 130}$ pour les arbres de chaque placette en fonction de l'indice de productivité de référence $I_{I}$, déterminé par le peuplement de futaie de chaque couple (cf. 2.21.). On peut ainsi remarquer que la dispersion des valeurs de $\mathrm{C} 130 / \widehat{C} \widehat{130}$ peut varier de façon importante pour des placettes dindice $I_{F}$ comparable, par exemple pour les couples 2 et 4 ou 8 et 9 . L'examen, simultanément, des distributions des valeurs de C $130 / \widehat{C} \widehat{130}$, réalisé selon le procédé décrit à la figure 4 , montre de plus une hétérogénéité de la forme de la distribution (normale, dissymétrique à gauche ou à droite) liée à l'échantillonnage réalisé dans chaque placette; dans un tel cas, la moyenne des valeurs observées (ici équivalente à $I \bar{v}$ ), n'est pas le meilleur descripteur de la distribution (cf. ANDERSON et al., 1974, pp. 88-92). La recherche d'un autre descripteur, davantage indépendant de l'échantillonnage réalisé et pouvant être identifié à un indice de productivité pour les taillis-sous-futaie, a porté sur des mesures de localisation de la distribution : valeur de $\mathrm{C} 130 / \widehat{C} \widehat{13} 0$ au-dessus de laquelle se trouve un pourcentage donné de valeurs observées (17). Différents indices ont ainsi été calculés pour lesquels on observait $10,20,30,40$ et 50 p. 100 de valeurs de $\mathrm{C} 130 / \widehat{C} \widehat{130}$ supérieures : les valeurs d'indice ainsi obtenues pour chaque placette ont été observées conjointement avec l'indice de productivité de référence $I_{k}$ de chaque couple. Les graphiques corresponçants (non reproduits ici), font apparaître à chaque fois une relation linéaire entre les deux indices démontrant la validité de la mesure proposée pour identifier la productivité relative des taillis-sous-futaie (ce résultat était déjà apparent sur la figure 10 où ont été figurées les médianes de chaque distribution). La mesure de localisation retenue pour la distribution des valeurs de $\mathrm{C} 130 / \widehat{\mathrm{C} 130}$ a été choisie pour qu'elle minimise lexpression $S^{2}(a, b)$, telle que :

$$
S^{2}(a, b)=\sum_{j=1}^{14}\left\{\left[(C 130 / C \widehat{13} 0)_{t}\right]_{j}-\left[a+b \mathbf{I}_{k}\right]_{j}\right\}^{2}
$$

où $(\mathrm{C} 130 / \widehat{\mathrm{CT}} 30)_{\kappa}$ désigne la mesure de localisation telle que $\alpha$ p. 100 des valeurs de la distribution soient supérieures à $(\mathrm{C} 130 / \mathrm{C} \widehat{30})_{k}$. La valeur de $\alpha$ minimisant $S^{2}(a, b)$ a été trouvée égale à 30 p. 100 et le graphique correspondant

(16) On aurait en effet, en faisant la somme des valeurs $I^{j} v_{i}^{j}$ pour les $n$ arbres de la placette $j$ et en divisant cette somme par $n$ :

$$
\frac{1}{n} \sum_{i=1}^{n} I^{i} v_{i}^{j}=I^{j} \sum_{i=1}^{n} \frac{v_{i}}{n}=I^{i} \frac{n \bar{v}^{j}}{n}=I^{j} \bar{v}^{j}=I^{j} V
$$

si la vigueur moyenne des $n$ arbres $\overrightarrow{\mathrm{v}}^{j}$ est égale à $V$ pour toutes les placettes.

(17) Cette mesure de Jocalisation revient à diviser le lot des observations ordonnées en pourcentages de valcurs observées : la valeur caractéristique pour laquelle on a a $\%$ d'observations supérieures à cette valcur (c'cst la médiane si $\alpha=50$ p. 100) peut être déterminée plus aisément en calculant les fréquences relatives cumulées de la distribution (pour plus de détails, voir ANDERSON et al., 1974, pp. 71-75). 
traduisant la relation linéaire entre l'indice de productivité ainsi déterminé pour les taillissous-futaie, soit $\mathrm{I}_{\mathrm{T} s \mathrm{~F}}$, et lindice de référence de la futaie $\mathrm{I}_{\mathrm{F}}$ apparaît à la figure 11 . Trois couples $(11,4$ et 16$)$ se sont individualisés quelle que soit la valeur choisie de $\alpha$ et ils ont été éliminés de l'analyse (bien qưils soient représentés sur la figure 11): pour le couple 11 , la distribution des valeurs de $\mathrm{C} 130 / \widehat{C} 130$ est très dissymétrique à gauche, et il se peut que les arbres les plus vigoureux aient été systématiquement abattus lors des exploitations; pour les couples 4 et 16 à distribution de $\mathrm{C} 130 / \widehat{C} \widehat{130}$ presque normale, on peut remettre en cause l'identité des conditions de fertilité de station pour la futaie et le taillis-sous-futaie de chaque couple.

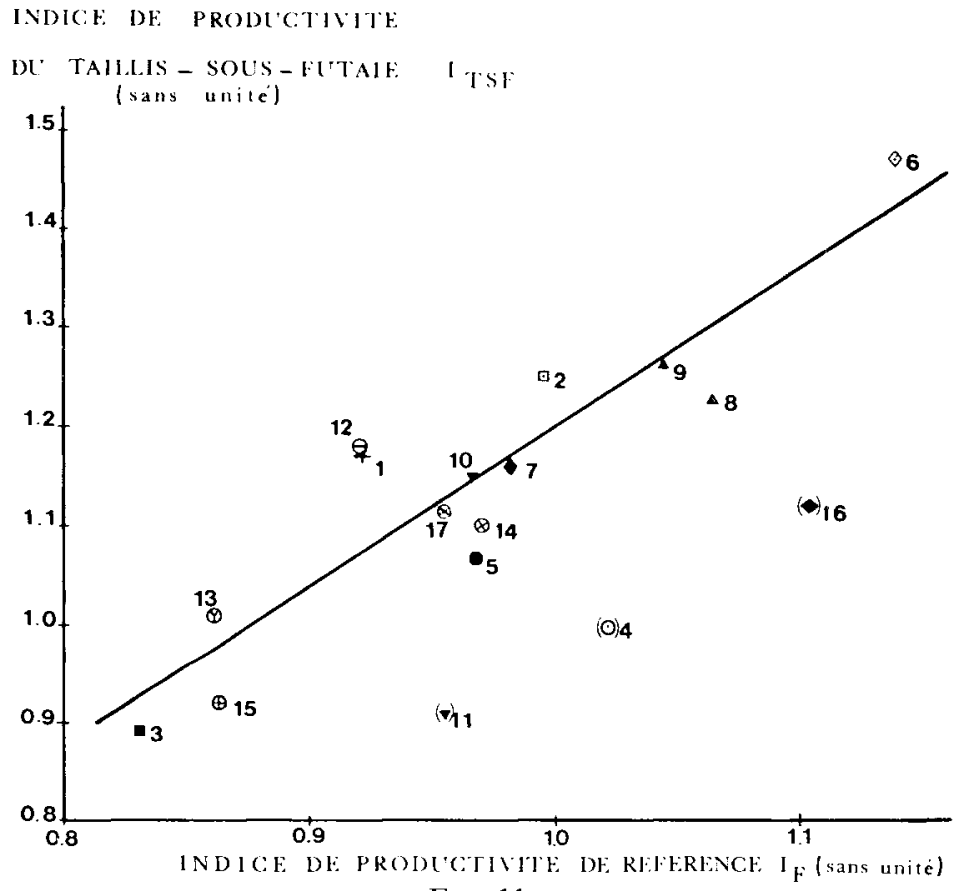

FIG. 11

Relation entre l'indice de productivité défini pour les taillis-sous-futaie (Irss) et l'indice de productivité de référence mesuré par la hauteur dominante relative de la futaie $\left(I^{\prime}\right)$ :

la relation linéaire établie ne tient pas compte des couples 11.4 et 16 jugés «aberrants» (voir le texte pour les explications données).

Relationship between the relative productivity index defined for the coppice-with-standards $\left(I_{T: i}\right)$ and the relative productivity index for the high forest $(I)$ : the linear relationship shown is established without pairs of plots 11,4 and 16 judged erratic.

L'indice de productivité du taillis-sous-futaie est défini comme suit : c'est la valeur prise par le rapport de la circonférence observée à la circonférence cstimće par la relation (1) de l'arbre pris dans un échantillon d'arbres parmi les plus vigoureux du taillis-sous-futaie et tel que $30 \%$ des arbres de l'échantillon aient une valeur de ce rapport supéricure à la sienne.

For the definition of $I_{F}$, see fig. 10. $I_{T S}$ is defined as follows : it is the value of the ratio of observed to estimated breast height girth [w'ith relation (l)] of the standard tree of a coppice-with standards, so as $30 \%$ of the values of the ratio for a sample of picked trees lie above it. 

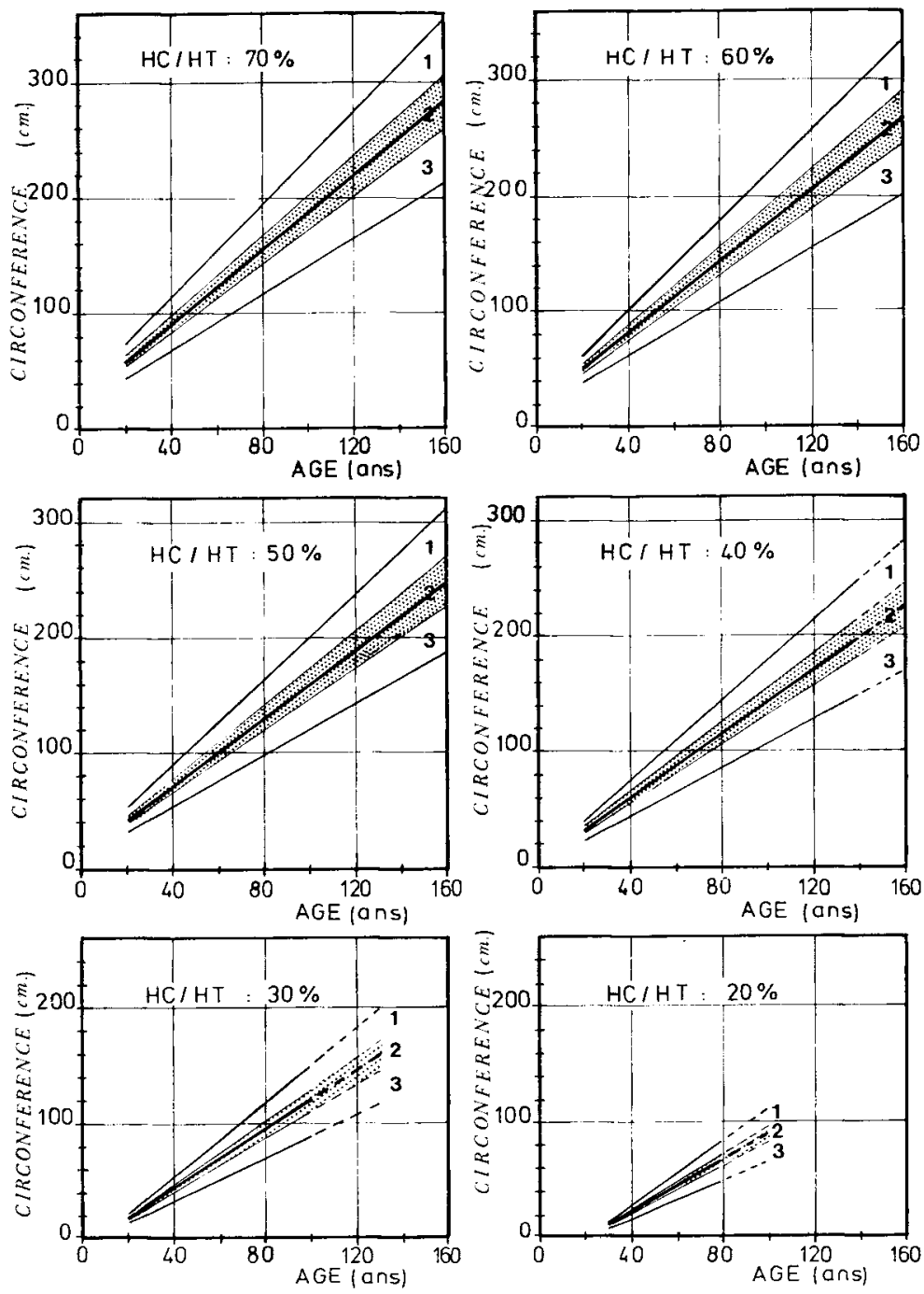

FIG. 12

Développenent hypothétique de la circonférence des réserves de taillis-sous-futaie dans des conditions de concurrence variables,

définies par la hauteur relative du houppier des arbres (variant ici de 20 à $70 \%$ ), et pour différents niveaux de productivité,

niveaux correspondant à ceux définis pour la futaie (cf. fig. 1).

Hypothetical development of the breast height girth of standard tres in coppice-with-standards for various values of the competitive status of trees (mean relative crown height varying from $20 \%$ to $70 \%$ ) and for three productivity classes identical to those defined in fig. 1.

Cette figure s'applique à des arbres dont la vigueur est équivalente à celle des arbres pris comme référence pour définir l'indice de productivité des taillis-sous-futaie (cf. fig. 11). Elle peut servir directement à estimer la classe de productivité des taillis-sous-futaie (voir Annexe pour des explications détaillées).

This figure applies to trees of the same vigour as those used as a reference for the definition of the relative productivity index for coppice-with-standards (see fig. 11); this figure can be used to identify the productivity class of a coppice-with-standards (see Appendix for explanations). 
Finalement, la remarque suivante peut être faite concernant la mesure de localisation de la distribution des valeurs de $\mathrm{C} 130 / \widehat{\mathrm{C} 30}$ retenue comme indice de productivité. Cette mesure consiste à prendre en considération la fraction des arbres de l'échantilion (30 p. 100) qui ont les valeurs de $\mathrm{C} 130 / \widehat{C} \widehat{13} 0$ les plus élevées, c'est-à-dire les 30 p. 100 qui sont de vigueur supérieure (arbres d'élite); lindice (C 130/ $\widehat{\text { T30 }}$ a sapplique alors à des arbres de vigueur comparable d"un peuplement de taillis-sousfutaie à lautre.

\section{Applications}

Deux applications peuvent être données à l'issue de cette étude : la première répond à l'objectif principal fixé, cest-à-dire la détermination d'un indice de productivité pour les taillis-sous-futaie de chêne, tel quil a été défini atl paragraphe précédent. La deuxième application concerne la possibilité cestimer lindice de productivité équivalent pour un peuplement de futaic (tel que défini en 2.2.); en effet, la relation linćaire entre ces deux indices, décrite à la fígure 11, peut être exprimée sous la forme:

$$
I_{F}=a^{\prime}+b^{\prime} I_{T \ll F}
$$

Lestimation par les moindres carrés des paramètres de la relation (4) a domné : $a^{\prime}=0.3729 ; b^{\prime}=0.5171$ (coefficient de corrélation $R^{2}=0.827$ ).

La ligure 12 peut constituer alors une synthèse des résultats obtenus : le développement de la circonférence de réserves de taillis-sous-futaie, de vigueur équivalente à celle des arbres pris comme référence pour définir lindice de productivité, répond aux différents schémas représentés lorsque le statut concurrentiel des arbres (mesuré par la hauteur relative du houppier) varie el lossque changent les conditions de fertilité de la station : les trois niveaux de prociuctivité figurés sont équivalents, pour la futa:e, à ceux de la ligure 1 et la zone en pointillé correspond de la même façon au niveau de produclivité de la classe unique de la table de production de PARDÍ pour le chêne en secteur ligérien.

Cette figure permet également la déiermination directe de la classe de productivité d'un taillis-sous-futaie donné à partir de la mesure d’un échantilon d'arbres (circonférence, âge, hauteur relative du houppier) choisis parmi les plus vigoureux des réserves de taillis-sous-futaic (tel qu'il a été procédé pour cette étude); il suffit pour cela de figurer les points représentatifs de chaque arbre sur le schéma voulu de la figure 12, dépendant de la valeur de HC/HT : le peuplement de taill:s-sous-futaic appartiendra ainsi à celle des 3 classes de productivité $(1,2$ ou 3$)$ figurées pour laquelle la fréquence relative cumulée des valcurs de classe ordonnées (par productivité croissante) sera

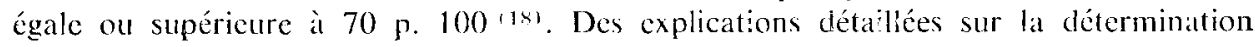

(18) La classe de productivté du taillis-sous-futaie n'est pas la moyenne des valeurs de classe donnéc par les aubres de l'échantillon, à partir de la ligure 12 : celle-ci concerne ell effet des arbres de vigueur bien délcrminée, ceux servant de référence pour définir l'indice de productivité et ici la classe de productivité. On peut alors montrer que prendre la valeur de classe déterminéc à partir de la figure 12 , et telle que $30 \% .100$ des valeurs de classe observées pour J'échantillon soient supérieures ou égales à celle-ci, revient bien à baser la classe de productivité sur des arbres de même vigueur que ceux considérés à la figure 12. 
de la classe de productivité d'un taillis-sous-futaie figurent en Annexe de cet article, et un cas concret y est traité. L'estimation précise de l'indice de productivité du taillissous-futaie, basé sur la distribution des valeurs de C $130 / C \widehat{130}$ de l'échantillon d'arbres, fait également l'objet d'un exposé détaillé en Annexe et du même cas concret d'application.

La détermination de la classe de productivité à partir de la figure 12 fournit immédiatement la classe de productivité équivalente d'un peuplement de futaie; la relation (4) permet quant à clle d'estimer l'indice de productivité de la futaie correspondant à celui du taillis-sous-futaie, indice que l'on peut alors exprimer en termes de hauteur cominante du peuplement de futaie à l'âge de référence de 160 ans.

Ayant ainsi déterminé le niveau de productivité d'un taillis-sous-futaie, équivalent à celui d'une futaie sur station de fertilité comparable, il est possible de prévoir de manière approchée la productivité en volume qu'aurait ce peuplement s'il était converti en futaie en se réferrant à la table de production de PArdé (par exemple : $5,8 \mathrm{~m}^{3 / \mathrm{ha}} / \mathrm{an}$ à 160 ans pour le niveau de productivité correspondant à celui de la table). II convient de noter aussi que la productivité en volume du taillis-sous-futaie, bien que plus difficile à évaluer, doit elle-même pouvoir être reliée à l'indice de productivité défini ici pour les taillis-sous-futaie : DE LEMPs a pu ainsi estimer pour les quatre types de stations de Perrin (cf. fig. 3) l'accroissement en volume des réserves du taillis-sous-futaie au cours d'une révolution de 25 ans, pour une composition normale donnée; on constate au tableau suivant que l'accroissement relatif du volume est bien lié au niveau de productivité (mesuré ici par l'accroissement moyen annuel sur le rayon des arbres d'élite parvenus à l'âge d'exploitabilité).

\begin{tabular}{c|c|c|c|c|c}
\hline $\begin{array}{c}\text { Type de } \\
\text { station }\end{array}$ & $\begin{array}{c}\text { Accroissement } \\
\text { moyen } \\
\text { sur le rayon } \\
\text { (arbres d'élite }(\mathrm{mm})\end{array}$ & $\begin{array}{c}\text { Volume } \\
\text { tige (1) } \\
\text { initial } \\
\left(\mathrm{m}^{3} / \mathrm{ha}\right)\end{array}$ & $\begin{array}{c}\text { Volume } \\
\text { tige }(\mathbf{1}) \\
\text { final } \\
\left(\mathrm{m}^{3} / \mathrm{ha}\right)\end{array}$ & $\begin{array}{c}\text { Accroissement } \\
\text { en volume } \\
\left(\mathrm{m}^{3} / \mathrm{ha}\right)\end{array}$ & $\begin{array}{c}\text { Accroissement } \\
\text { relatif } \\
\text { du volume }\end{array}$ \\
\hline 1 & $-3,1$ & 45 & 102,7 & 57,7 & 0,825 \\
2 & 3,2 & 40 & 85 & 45 & 0,754 \\
3 & 2,9 & 45 & 93 & 48 & 0,726 \\
4 & 2,2 & 40 & 80 & 40 & 0,693 \\
\hline
\end{tabular}

(1) Volume tige $=$ volume découpe bois d'ouvre, sur écorce.

Les résultats de cette étude permettent enfin de micux préciser, au moment où l'on parle de sylviculture à larges cspacements pour le chêne, le gain attendu sur laccroissement moyen radial des tiges quand on maintient les arbres à différents niveaux de concurrence. La figure 12 fournit ainsi pour des stations de fertilité équivalente à celle de la table de PARDÉ (il s'agit d'arbres d'élite) : 


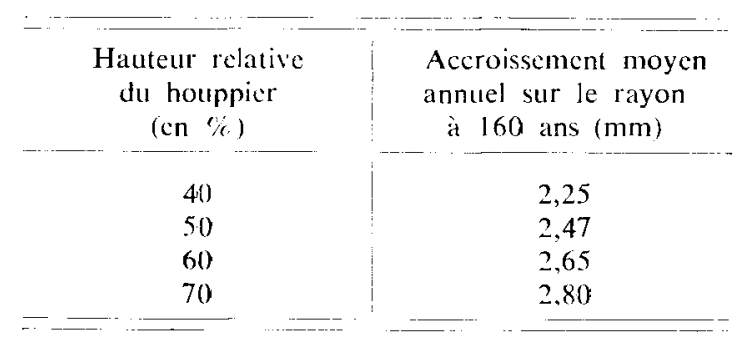

L'augmentation de laccroissement moyen annuel radial quand le niveau de concurrence diminue reste assez faible $19 \%$. L 'intérêt de maintenir les arbres dans des conditions de densité de peuplement faibles résiderait davantage dans la diminution de l'âge d'exploitabilité : un arbre de $60 \mathrm{~cm}$ de diamètre peut être récolté à 110 ans (classe de fertilité moyenne) si il est maintenu avec une hauteur relative du houppier de 70 p. 100 , à près de 140 ans seulement si celle-ci n'est que de 40 p. 100 . Mais d'autres facteurs peuvent intervenir, telle la longueur du fût qui diminue lorsque $\mathrm{HC} / \mathrm{HT}$ augmente ou la décroissance de la tige, plus forte dans ces mêmes conditions.

\section{Conclusion}

A partir de données recueillies à un moment donné sur des arbres pris dans les différentes classes d'âge de peuplements de taillis-sous-futaie, il a été possible d'en déduire le développement caractéristique de la circonférence au cours du temps dépendant des conditions de concurrence dans lesquelles ont poussé les réserves des taillissous-futaie, conditions appréciées par la hautcur relative actuelle du houppier des arbres. Il a été possible ensuite, se basant sur deux hypothèses, proportionnalité des courbes de croissance en circonférence à la vigucur des arbres d'une part, à la fertilité de la station d'autre part, de déduire de la relation établie entre la circonférence, l'âge et la hauteur relative du houppier, un indice de productivité pour les taillis-sous-futaie de chêne: l'évaluation de la productivité relative des stations concernées par une mesure indépendante et couramment utilisée, hauteur dominante diun peuplement de futaic à un âge déterminé (2(1), a permis de s’assurer de la validité de la mesure proposée pour les taillis-sous-futaie et davo:r une mesure moins dépendante de l'échantillonnage réalisé en se réferrant à des arbres de vigueur comparable.

Ces résultats devraient pouvoir être vérifiés dans le cadre d'une application à partir de mesures similaires à celles réalisées ici dans quelques couples futaic/taillis-sous-futaie nouveaux. Une autre vérification consisterait à étudier l'effet des conditions de station (sol, topographie, climat) sur la productivité du chêne à partir d'évaluations de la productivité faites en futaie et en taillis-sous-futaie.

(19) A titre de comparaison, l'accroissement moyen sur le rayon à 160 ans des arbres de la placette de futaic du couple 7 (même productivité que celle de la Table de production de Pardé) est le suivant : $1,66 \mathrm{~mm}$ pour larbre de surface terrière moyenne des 100 plus gros à l'hectare, $1,88 \mathrm{~mm}$ pour l'atbre de surface terrière moyenne des 10 plus gros à l'hectare.

(20) Ici, en fáit, la productivité relative de la futaie est mesurée directement par un indice, rapport de la hauteur dominante de la futaie à la hauteur dominante au nême âge donnéc par la table de production pour les futaies de chêne du Centre. 
La recherche d'un indice de productivité fiable pour des peuplements inéquiennes et de structure variable comme ces taillis-sous-futaie a nécessité des considérations concernant en particulier l'effet de la concurrence et de la vigueur des arbres sur leur croissance. Elle devrait permettre d'étendre la méthodologie employée à des peuplements de structure aussi éloignée de celle des peuplements purs et équiennes que ceux étudiés ici.

Des indications peuvent être déduites de cette étude concernant la productivité en volume attendue de peuplements de taillis-sous-futaie qui seraient convertis en futaic de densité proche de celle de la table de production établie pour le chêne dans cette région. Ces conditions de sylviculture ne peuvent cependant concerner que des massifs assez importants et de bonne productivité ; les conditions de croissance du chêne traité en peuplements moins denses et donc à âge d'exploitabilité plus jeune doivent être mieux étudiées : ces conditions existent dans les taillis-sous-futaie, et on peut se demander comme De Lemps si ce traitement n'est pas à nouveau à redécouvrir, tout du moins pour la forêt privée, et s'il n'a pas pâti de n'être pas suivi rigoureusement par le contrôle du matériel à laisser sur pied grâce à des normes adaptées à la fertilité des stations.

\section{Remerciements}

1) La collaboration du Centre régional de la Propriété forestière de la région Centre (MM. J. Doussineau, M. Formery) a permis, grâce à une prospection sur le terrain, la détermination d'un nombre suffisant de couples futaie/taillis-sous-futaic, base de cette étude. Elle a facilité également les relations avec les propriétaires forestiers particuliers.

2) L'aide de l'Office National des Forêts (Centres de Gestion de Blois, Montargis et Bourges : MM. R. Lafouge, J. Hubert et Y. Richer de Forges) nous a également été précieuse pour la détermination de certains couples futaie/taillis-sous-futaie.

3) Les analyses graphiques nécessaires pour cette étude ont été grandement facilitées grâce à un programme informatique (sur le mode conversationnel) conçu par J.-M. OTTORINI à la Station de Sylviculture et de Production et permettant d'analyser les données selon les principes exposés au paragraphe 4 de cet article.

4) Au cours de ce travail, il a également été fait large usage de la programmathèque de la Station de Biométrie du C.N.R.F. installée sur un ordinateur Mini 6 et dont un manuel de présentation et d'utilisation «Amance 81 » a été édité. L'existence de plusieurs terminaux (écrans notamment) a grandement facilité les examens graphiques cités précédemment.

5) La réalisation des figures de cet article est due à L. GnRros, technicien à la Station de Sylviculture et de Production, la dactylographie et la mise en page du manuscrit à R. Gross de cette même Station.

6) Suivarst la suggestion des lecteurs du manuscrit, N. Decourt et M. HubliRT, ainsi que celle de H. Oswald, une Annexe a été ajoutée à la suite de cet article à l'intention des personnes susceptibles d'utiliser les résultats de ce travail dans le cadre d'une application.

\section{Summary \\ A measure of relative productivity for oak coppice-with-standards in the Center region of France.}

The aim of this study is to know and measure capability for growth and production of oak of sites actually covered with stands treated as oak coppice-with-standards and often poor with standards in the Center region of France. Seventeen pairs of plots « high forest/ coppice-with-standards » have been established : each pair of plots includes one plot in an oak coppice-with-standards and the other one in an oak high forest, both in the same site conditions. 
The high forest plot of each "pair» gives a reference index of productivity for oak : it is defined as the ratio of top height of the stand to top height at the same age given by the single fertility class yield table for oak high forests of the Center region. Three procluctivity classes have been determined for the seventeen pairs of plots, top height at age 160 varying from 28 to 36 meters.

The research of and index of productivity for oak coppice-with-standards has been bascd on the study of gith development of the standards (previous results had shown a good relationship with fertility of sites). Other factors of girth growth have been considered : past competitive conditions for the standards synthesized by the actually measured ratio of crowr height to total height of 1rees, individual variability of trees (in particular by sampling "picked trees $»$ in the population of the standardis of each coppice-with-standards).

A relationship between breast height girth of the standards, age and relative crown height of trecs has been established. The productivity index for oak coppice-with-standards has then been identified as a particular measure of the distribution of valucs of the ratio of observed to estimated girth (tormer relation) of sampled standards in each coppice-withstandards plot.

A simple linear relationship has been established between the productivity index for oak coppice-with-standards and the productivity index for oak high forests. It allows comparison of relative productivity of sites with the one or the other type of oak stand. It allows also to predict volume productivity of known relative productivity oak coppicewith-standards which would be converted to high forests stands, with the help of the yield table for oak high forests of the Center region.

Practical application of the results of this study, that is estimation of the index of productivity of oak coppice-with-standards, received particular attention : people interested will find in Appendix, with a definite example. all details on the way to use basic relations and figures necessary for this estimation.

Reçu pour publication le 5 août 1982. Accepté pour publication le 13 janvier 1983.

\section{Références bibliographiques}

Anderson T.W., Sciove: S.L., 1974. Introductory statistical analysis. Houghton Mifflin Company.

BAR'TET E., 1891. Recherches sur le mode d'accroissement des chênes de taillis-sous-futaic. Rev. Eaux Forêts, sept. 1891, 393-405.

Becker M., 1979. Une étude phyto-écologique sur les plateaux calcaires du Nord-Est (Massif de Haye - 54). Utilisation de lanalyse des correspondances dans la typologie des stations. Relations avec la productivité ct la qualité du hêtre el du chêne. Ann. Sci. For., 36 (2), 93-124.

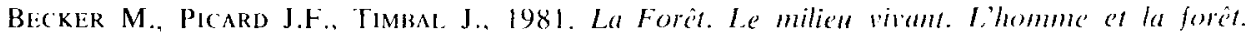
Les grandes forêts de France. Les arbres de la forêt. Masson et Réalisations Editoriales Pédalgogiques, Paris.

Carmean W.H., 1975. Forest site quality evaluation in the United States. Adv. Agron., 27, 209-269.

Curtin R.A., 1970. Dynamics of tree and crown structure in Eucalyptus obliqua. For. Sci., 16, (3), 1970.

Draper N.R. SmtTh H., 1966. Applied regression andysis. John Wiley and Sons. Inc. New York.

Le Goff N., Ottorini J.M., 1979. Normes de densité pour les hêtraies du Nord-Est et du Nord-Ouest de la France. Ann. Sci. For., 36 (4), 281-298.

Le Goff N., 1982. Productivité du Frêne en région Nord-Picardie. A. - Courbes de croissance en hauteur. Ann. Sci. For., 30 (3), 259-287. 
De Lemps F., 1951. Volume critique, plan de balivage et composition normale dans les taillis-sous-futaie. Rev. For. Fr., n* 9, 552-572.

Mosthller F., Tukey J.W., 1977. Data analysis and regression, A second conrse in staristics. Addison-Wesley Publi. Cy.

Ottokini J.-M., Nys C., 1981. Application des données de l'I.F.N. à l'étude de la production du Pin sylvestre en Margeride. A. - Etude de la croissance en hauteur. Ann. Sci. For., 38 (2), 223-236.

OTIORINi J.-M., 1981. Application des données de li.F.N. à l'étude de la production du Pin sylvestre en Margeride. B. - Etude de la production totale en volume. Amn. Sci. For., 38 (4), 487-502.

PARDÉ J., 1962. Table de production pour les forêts de Chêne rouvre de qualité tranchage du secteur ligérien. Notes Techniques Forestières. Mars 1962, Note $\mathrm{n}^{\circ} 11$. Station de Recherches et Expériences Forestières, Nancy.

Perrin H., 1939. Sur l'accroissement des chênes de taillis-sous-futaie. Rev. Eaux Forêts, avril 1939.

\section{Annexe \\ Détermination de la productivité relative d'un taillis-sous-futaie (exemple traité avec les données du couple 16)}

\section{Echantillonnage et mesures}

Les arbres constituant l'échantillon sont choisis après inventaire complet des circonférences des réserves de taillis-sous-futaie (à partir de la dimension de baliveau) sur une placette de surface comprise entre 0,5 et 1 hectare; les valeurs de circonférence sont reportées tel quindiqué à la figure 4 et les classes d’âge (supposées) délimitées. Les arbres à mesurer, au nombre de 5 par classe d'âge si possible, seront choisis parmi ceux de circonférence égale ou supérieure à la valeur médiane des circonférences de chaque classe d'âge (arbres «d'élite »).

Les arbres de l'échantillon feront alors lobjet des mesures suivantes :

- circonférence (C 130) mesurée au ruban à $1,30 \mathrm{~m}$ de hauteur.

- hautcur totale (HT) de la base de l'arbre au bourgeon terminal,

- hautcur du houppier (HC) de la base du houppier au bourgeon terminal (voir paragraphe 3.2. du texte pour définition précise),

- âge total,

on en déduira la hauteur relative du houppier (HC/HT), rapport (exprimé en \%) de la hautcur du houppier à la hauteur totale.

Les données obtenues pour le couple 16 figurent au tableau 3 (trois premières colonnes).

\section{Evaluation de la productivité relative du taillis-sous-futaie}

Deux modes d'évaluation de la productivité relative peuvent alors être suivis :

- évaluation rapide par la détermination de la classe de productivité à l'aide d'une abaque conforme à cel'e de la figure 12 ,

- évaluation plus précise par la détermination de l'indice de productivité en utilisant la relation (3) (ce qui nécessite de disposer d'unc petite machine à calculer). 


\subsection{Classe de productivité du taillis-sous-futaie}

Les abaques de la figure 12 présentent les "plages» de circonférence pour les trois classes de productivité distinguées, suivant le statut concurrentiel des arbres (mesuré par la hauteur relative du houppier). Elles concernent cependant des arbres de vigueur bien déterminée (cf. paragraphe 6). La classe de productivité du taillis-sous-futaie sera alors obtenue de la façon suivante :

- détermination d'une valeur de classe pour chaque arbre de l'échantillon, en reportant sur l'abaque voulue de la figure 12 (dépendant de la valeur de $\mathrm{HC} / \mathrm{HT}$ ) le point représentatif (C 130, Age) de l'arbre considéré. Les données correspondantes pour lexemple traité figurent au tableau 3 , colonne 4 ;

- calcul des fréquences relatives cumulées des valeurs de classe des arbres, ordonnées par classe de productivité croissante.

La classe de productivité du taillis-sous-futaie est celle pour laquelle la fréquence relative cumulée des valeurs de classe ordonnées des arbres égale ou dépasse 70 p. 100.

Le tableau suivant présente les résultats obtenus pour l'exemple traité. à partir des données de la colonne 4 du tableau 3 :

\begin{tabular}{c|c|c|c}
$\begin{array}{c}\text { Classes } \\
\text { de productivité } \\
\text { ordonnéss }\end{array}$ & $\begin{array}{c}\text { Fréquence } \\
\text { des valeurs } \\
\text { de classe }\end{array}$ & $\begin{array}{c}\text { Fréquence cumulée } \\
\text { des valeurs } \\
\text { de classe }\end{array}$ & $\begin{array}{c}\text { Fréquence relative } \\
\text { cumulée des valeurs } \\
\text { de classe (en \%) }\end{array}$ \\
\hline 3 & 13 & 13 & 61,9 \\
2 & 7 & 20 & 95.9 \\
1 & 1 & 21 & 100 \\
\hline
\end{tabular}

Le taillis-sous-futaie du couple 16 se situe ainsi sur une station de classe de productivité 2 car moins de 70 p. 100 des arbres indiquent une classe $3(61,9$ p. 100) et plus de 70 p. 100 indiquent la classe 2 ou $3(95,9$ p. 100).

Un peuplement de futaic de chêne sur cette station aurait donc une productivité comparable à celle des peuplements de la table de production de PARDé (correspondance entre les trois classes de productivité définies pour le taillis-sous-futaic et celles définies pour les peuplements de futaie).

\subsection{Indice de productivité du taillis-sous-futaie $\left(I_{T S \mathrm{H}}\right)$}

La relation (1) - cf. paragraphe 4 du texte - appliquée aux arbres de l'échantillon permet d'estimer la circonférence attendue $\widehat{C} \widehat{130}$ de ces arbres, compte tenu de leur âge et de la hauteur relative du houppier, pour les conditions de fertilité moyenne des stations étudiées (colonne 5 du tableau 3 pour lexemple traité).

Le rapport de la circonférence observée $\mathrm{C} 130$ à la circonférence estiméc C $\widehat{\mathrm{B}} \mathbf{3}$ ) des arbres de l'échantillon (colonne 6 du tableau 3) est à la base de la mesure de la productivité relative du taillis-sous-futaie : lindice de productivité est en effet défini 
comme la valeur du rapport $\mathrm{C} 130 / \mathrm{C} \widehat{130}$ au-dessus de laquelle se siluent 30 p. 100 des valeurs de $\mathrm{C} 130 / \widehat{\mathrm{C} 130}$ observées pour léchantillon.

Cette mesure de localisation de la distribution des valeurs de C $130 / C \widehat{130}$, notée (C 130/C T30) cians le texte (cf. paragraphe 5), peut être effcetuée plus faciiement en regroupant les valeurs de $\mathrm{C}$ i $30 / \widehat{\mathrm{C}} 30$ par classes : une valeur aporochée de (C 130/C $\widehat{130})_{1,}$ est alors obtente en calculant lexpression suivante :

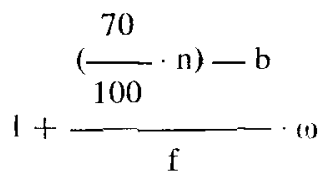

(d'après ANDi:RSON at al. ,1974, pp. 94-97).

où :

$n=$ nombre doobservations (arbres échantillonnés).

$1=$ va:eur inféricure de la ciasse de $\mathrm{C} 130 / \widehat{C 130}$ contenant $(\mathrm{C} 130 / \mathrm{C} \widehat{130})_{t}$, cestà-dire pour laquelle la fréquence relative des valeurs observées de $\mathrm{C} 130 / \mathrm{C} \widehat{130}$ égale ou dépasse $70 \%$. $\widehat{C} \widehat{130})_{\omega}$.

$\mathrm{f}=$ fréquence des valeurs de $\mathrm{C} 130 / \mathrm{C} \widehat{30}$ dans la classe contenant (C 130/

$\mathrm{b}=$ fréquence cumulée des valeurs de $\mathrm{C} 130 / \mathrm{C} \widehat{\mathbf{1 3}} 0$ pour loutes les classes inférieures à celle contenant $(\mathrm{C} 130 / \mathrm{C} \widehat{130})_{\alpha}$.

$\omega=$ largeur de lintervalle des classes de C $130 / \mathrm{C} \widehat{130}$.

Le nombre de classes de C 130/ $\widehat{130}$ - done aussi lintervalle des classes peut être fixé d'après le nombre $n$ d’observations. En pratique, $n$ devrail être compris entre 10 et 25 (5 arbres mesurés par classe d’âge, 2 à 5 classes dâge pour les peuplements de tailis-sous-futaie de cette étude) : pour un tel effectif, il est suggéré de faire 5 classes pour regrouper les observations (cf. ANDERSON at al., 1974, p. 97).

La détermination de l'indice de productivité du taillis-sous-futaic du couple 16 servira à illustrer le mode de calcul de $(\mathrm{C} \mathrm{130/ \widehat {C } \widehat { 1 3 0 } )})_{\iota}$ qui vient d'être présenté. A partir des données du tableau 3 (colonne 6), les valeurs de C $130 /$ C $\widehat{130}$ pour l'échantillon d’arbres peuvent être présentées de la façon suivante :

\begin{tabular}{c|c|c|c}
\hline $\begin{array}{c}\text { C } 130 / \mathrm{C} \widehat{130} \\
\text { (classes) }\end{array}$ & $\begin{array}{c}\text { Fréquence } \\
\text { des classes }\end{array}$ & $\begin{array}{c}\text { Fréquence } \\
\text { cumuléc }\end{array}$ & $\begin{array}{c}\text { Fréquence relative } \\
\text { cumuléc }\end{array}$ \\
\hline $0,7-0,9$ & 5 & 5 & $-23,8$ \\
$0,9-1,1$ & 9 & 14 & 66,7 \\
$1,1-1,3$ & 6 & 20 & 95,2 \\
$1,3-1,5$ & 0 & 20 & 95,2 \\
$1,5-1,7$ & 1 & 21 & 100 \\
\hline
\end{tabular}




\section{TABLenU 3}

Donneses dendrométriques relatives à l'échantillon

des réserves de taillis-sons-futaie du couple 76.

nécessaires à l'estimation de la productivitó relative du taillis-sons-futaie.

Tree measures for the sample of standards of coppice-with-standards from plot $\mathrm{n}^{\circ} \mathrm{16}$, and necessary for the estimation of its index of relative productivity.

\begin{tabular}{|c|c|c|c|c|c|}
\hline $\begin{array}{l}\text { C 130) } \\
(\mathrm{cm})\end{array}$ & $\begin{array}{c}\text { Age lotal } \\
\text { (ans) }\end{array}$ & $\begin{array}{c}\mathrm{HC} / \mathrm{HT} \\
(\%)\end{array}$ & $\begin{array}{l}\text { Classe 11 } \\
\text { de productivité } \\
\text { (d'après } \\
\text { la figure 12) }\end{array}$ & 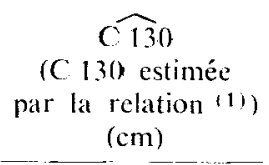 & $\frac{C 130}{C \widehat{130}}$ \\
\hline 35 & 24 & 56 & 3 & 44.5 & 0,787 \\
\hline 45 & 2.7 & 57 & 3 & 49,1 & 0,916 \\
\hline 43 & 23 & 74 & 3 & 54,5 & 0,790 \\
\hline 48 & 23 & 78 & 3 & 56,6 & 0,848 \\
\hline 50 & 26 & 62 & 3 & 51.2 & 0,977 \\
\hline 75 & 51 & 48 & 3 & 71,9 & 1,043 \\
\hline 70 & 50 & 62 & 3 & 82,3 & 0,850 \\
\hline 77 & 50 & 50 & 3 & 71.9 & 1.071 \\
\hline 80 & 35 & 49 & 1 & 52.9 & 1.512 \\
\hline 89 & 49 & 59 & 2 & 79.3 & 1,122 \\
\hline 112 & 84 & 72 & 3 & 135.0 & 0,830 \\
\hline 122 & 87 & 60 & 3 & 128,5 & 0,949 \\
\hline 148 & 70 & 77 & 2 & 120.1 & 1.2 .32 \\
\hline 132 & 76 & 62 & 2 & 116.4 & 1,134 \\
\hline 145 & 109 & 63 & 3 & 159.9 & 0.907 \\
\hline 160 & 111 & 66 & 3 & 166,1 & 0.963 \\
\hline 168 & 93 & 61 & 2 & 137,5 & 1,222 \\
\hline 187 & 111 & 79 & 3 & 178.1 & 1.050 \\
\hline 199 & 118 & 62 & 2 & 170,8 & 1.165 \\
\hline 202 & 117 & 78 & 2 & 185,7 & 1,088 \\
\hline 226 & 118 & 67 & 2 & 175,8 & 1,285 \\
\hline
\end{tabular}

(1) Troí classes de productivité ont été définies pour les taillis-sous-futaic de chêne en région Centre (classes 1, 2, 3 de la figure 12). Les points représentatifs (C 130, Age) des arbres. sur la figure 12. peuvent se situer en dehors des limites des classes: on alfecteria alors lat classe 0 aux arbres dont la circonférence excède la valcur supérieure de la classsc l au même âge el on affectra la classe 4 aux arbres dont la circonférence natteint pas la valeur inféricure alu même âge de la classe 3.

La classe contenant $(\mathrm{C} 130 / \mathrm{C} \widehat{130})$ " est la classe $1,1-1,3$ car c'est dans cette classe que la fréquence relative cumulée de $70 \mathrm{p} .100$ pour les valeurs de $\mathrm{C} 130 / \widehat{\mathrm{C13}} \mathrm{y}$ est atteinte. 
On a alors pour lexemple traité :

$$
\begin{gathered}
\mathrm{I}=1,1 ; \mathrm{n}=21 ; \mathrm{b}=14 ; \mathrm{l}=6 ;(1)=0,2 . \\
\mathrm{I}_{\mathrm{TSF}}=(\mathrm{C} 130 / \mathrm{C} \widehat{130})_{\alpha}=1,1+\left(\frac{\left(\frac{70}{100} \times 21\right)-14}{6}\right) 0,2 \\
\mathrm{I}_{\mathrm{TSSP}}(\text { couple } 16)=1,123
\end{gathered}
$$

- L’indice de productivité équivalent pour un peuplement de futaic, $I_{F}$, peut alors être estimé à l'aide de la relation (4) :

$$
\mathrm{I}_{\mathrm{F}}=0,3729+0,5171\left(\mathrm{I}_{\mathrm{T} \times \mathrm{F}}\right)
$$

soit, pour l'exemple traité : $\mathbf{I}_{\mathrm{r}}=0,954$.

L'indice $I_{F}$ peut lui-même être converti en hauteur dominante du peuplement de futaie à 160 ans (cf. 2.2.), soit $\mathrm{H}_{0}(160)$ :

$$
H_{0}(160)=32,0 \times I_{1} \text { (en mètres) }
$$

ce qui donne pour l'exemple traité $H_{0}(160)=30,53 \mathrm{~m}$ (la valeur ainsi estimée de la hauteur dominante du peuplement de futaie du couple 16 est sensiblement inférieure à la valeur récllement mesurée pour ce couple - cf. tableau 1 - ; il semble que la futaie se situe en fait sur une station de fertilité supérieure à celle du taillis-sous-futaie - cf. paragraphe 5 du texte).

Compte tenu de l'hétérogénéïté de structure des peuplements de taillis-sous-futaic utilisés dans cette étude, il est permis de penser que la méthode d'estimation de la productivité relative des taillis-sous-futaie de chêne présente une bonne fiabilité et donne de bons résultats dans la plupart des cas. Cependant un cas particulier peut se présenter, celui où la réserve du taillis-sous-futaie a été surexploitée (el notamment les arbres d'élite : cas vraisemblable du couple 11); dans ce cas, on sous-estimera la productivité réelle du taillis-sous-futaie (il semble qualors la distribution des valeurs de $\mathrm{C} 130 / \mathrm{C} \widehat{130}$ soit très dissymétrique : queue droite de la distribution très réduite ou même absente). 